\title{
The Structure of Reachable Sets and Geometric Optimality of Singular Trajectories for Certain Affine Control Systems in $\mathbb{R}^{3}$. The Sub-Lorentzian Approach
}

\author{
Marek Grochowski
}

Received: 3 May 2012 / Revised: 6 April 2013 / Published online: 10 December 2013

(C) Springer Science+Business Media New York 2013

\begin{abstract}
In this paper, we investigate the structure of reachable sets from a given point $q_{0}$ for a class of analytic control affine systems characterized, among other things, by possessing two singular trajectories initiating at $q_{0}$. The aim of the paper is to establish the connection between the minimal number of analytic functions needed for describing reachable sets and the number of geometrically optimal singular trajectories. The paper is written in a language of the sub-Lorentzian geometry. Also, the sub-Lorentzian geometry methods are used to prove theorems.
\end{abstract}

Keywords Sub-Lorentzian manifolds · Geodesics · Reachable sets · Geometric optimality $\cdot$ Vector distributions · A Martinet surface

Mathematics Subject Classifications (2010) 53B30 · 34H05 - 49K99

\section{Introduction}

\subsection{Preliminaries}

This paper is a continuation of the research started in $[8,9]$ and devoted to the study on reachable sets for noncontact sub-Lorentzian structures on $\mathbb{R}^{3}$, as well as for affine control systems induced by them. Similarly as in $[8,9]$ our objective is to investigate the interrelation of the structure of reachable sets from a given point $q_{0}$ for the mentioned systems and geometric optimality of singular trajectories starting at $q_{0}$ or-speaking in the subLorentzian language-geometric optimality of timelike abnormal curves starting at $q_{0}$ (a

M. Grochowski $(\bowtie)$

Faculty of Mathematics and Science, Cardinal Stefan Wyszyński University, ul. Dewajtis 5,

01-815 Warsaw, Poland

e-mail: m.grochowski@uksw.edu.pl

M. Grochowski

Institute of Mathematics, Polish Academy of Sciences, ul. Śniadeckich 8, 00-950 Warszawa, Poland 
trajectory of a control system starting from a point $q_{0}$ is said to be geometrically optimal if it is contained in the boundary of the reachable set from $q_{0}$; cf. [1]). The paper is arranged in such a way that, in the first four sections, we develop the theory in the sub-Lorentzian setting, and Section 5 contains applications of the obtained results to control affine systems.

For all facts and notions from the sub-Lorentzian geometry, the reader is referred to the previous papers by the author (see [6] and its reference section; see also [10]). Here, we recall only those basic facts that are needed for stating the results. Let $M$ be a smooth manifold, and let $H$ be a smooth distribution on $M$ of constant rank. For a point $q \in M$ and an integer $k \in \mathbb{N}$, we define $H_{q}^{k}$ to be the linear subspace in $H_{q}$ generated by all vectors of the form $\left[X_{1},\left[X_{2}, \ldots,\left[X_{i-1}, X_{i}\right] \ldots\right]\right](q)$, where $X_{1}, \ldots, X_{i}$ are smooth (local) sections of $H$ defined in a neighborhood of $q$, and $i \leq k$. We say that $H$ is a bracket generating distribution if for every $q \in M$, there exists an $i=i(q)$ such that $H_{q}^{i}=T_{q} M$. Now, by a sub-Lorentzian structure (or a sub-Lorentzian metric) on a manifold $M$, we mean a pair $(H, g)$, where $H$ is a bracket generating distribution on $M$, and $g$ is a smooth Lorentzian metric on $H$. The triple $(M, H, g)$ is called $a$ sub-Lorentzian manifold. Take a point $q \in M$; a vector $v \in H_{q}$ is said to be timelike if $g(v, v)<0$, nonspacelike if $g(v, v) \leq 0$, and null if $g(v, v)=0$ but $v \neq 0$. A time orientation of $(M, H, g)$ is, by definition, a continuous timelike vector field on $M$. Suppose $(M, H, g)$ to be time-oriented by $X$ and let $v \in H_{q}$ be a nonspacelike vector. We say that $v$ is future directed if $g(v, X(q))<0$. A curve $\gamma:[a, b] \longrightarrow M$ is called horizontal if it is absolutely continuous, $\dot{\gamma}(t) \in H_{\gamma(t)}$ a.e. on $[a, b]$, and $\dot{\gamma}$ is square integrable with respect to some Riemannian metric on $M$. From now on, all curves are supposed to be horizontal. We will also use the following abbreviations: $t$. for "timelike," nspc. for "nonspacelike," and f.d. for "future directed." We say that a curve $\gamma:[a, b] \longrightarrow M$ is t.f.d. (resp. nspc.f.d., null f.d.) if so is $\dot{\gamma}(t)$ a.e. on $[a, b]$. Fix a point $q_{0} \in M$ and its neighborhood $U$. The (future) timelike (resp. nonspacelike, null) reachable set from $q_{0}$ relative to $U$ is defined to be the set of all points in $U$ that can be reached from $q_{0}$ by a t.f.d. (resp. nspc. f.d., null f.d.) curve entirely contained in $U$. They are denoted respectively by $I^{+}\left(q_{0}, U\right), J^{+}\left(q_{0}, U\right)$, and $N^{+}\left(q_{0}, U\right)$. In the general case, all we can say about reachable sets is that int $I^{+}\left(q_{0}, U\right) \neq \varnothing$, and that the three reachable sets have the same interiors and closures with respect to $U$. In order to be able to say something more, we need to make certain assumptions on $U$. To this end, let us notice that if $U$ is sufficiently small, then our sub-Lorentzian metric can be extended to a Lorentzian metric, say $\tilde{g}$, on $U$. So $U$ is said to be a normal neighborhood of $q_{0}$ if it is a convex normal neighborhood of $q_{0}$ with respect to $\tilde{g}$, and $U$ is contained in some other convex normal neighborhood of $q_{0}$ with respect to $\tilde{g}$ (see [8] for a constructive definition of normal neighborhoods). Now, if $U$ is a normal neighborhood of $q_{0}$, we know that $J^{+}\left(q_{0}, U\right)$ is closed with respect to $U$ and moreover $c l_{U}\left(\operatorname{int} I^{+}\left(q_{0}, U\right)\right)=c l_{U}\left(\operatorname{int} N^{+}\left(q_{0}, U\right)\right)=J^{+}\left(q_{0}, U\right)$, where $c l_{U}$ stands for the closure with respect to $U$. Note that unlike the Lorentzian case, the boundary $\tilde{\partial} J^{+}\left(q_{0}, U\right)$ (here and below, $\tilde{\partial}$ means the boundary with respect to $U$ ) may contain timelike curves starting from $q_{0}$. It can be proved [6] that such curves are abnormal curves for the underlaying distribution $H$ (see [11] for a definition); they are also Goh curves (cf. [1]), but we do not need this latter fact in this paper. Let $X_{0}, \ldots, X_{k}$ be an orthonormal frame for $(H, g)$ defined on an open set $U$. We define the so-called geodesic (sub-Lorentzian) Hamiltonian $\mathcal{H}: T^{*} U \longrightarrow \mathbb{R}$, by formula $\mathcal{H}(q, p)=-\frac{1}{2}\left\langle p, X_{0}(q)\right\rangle+\frac{1}{2} \sum_{i=1}^{k}\left\langle p, X_{i}(q)\right\rangle$ (it is possible to define $\mathcal{H}$ in a global and invariant way; see [6]). Now, a curve $\gamma:[a, b] \longrightarrow$ $U$ is said to be a Hamiltonian geodesic if it can be represented as $\gamma(t)=\pi \circ \Gamma(t)$ where $\pi: T^{*} M \longrightarrow M$ is the canonical projection and $\dot{\Gamma}=\overrightarrow{\mathcal{H}}, \overrightarrow{\mathcal{H}}$ being the Hamiltonian vector field corresponding to the function $\mathcal{H}$. Hamiltonian geodesics do not change their 
causal character; moreover, null f.d. Hamiltonian geodesics are [6] locally geometrically optimal. Finally, let $U$ be an open subset in $(M, H, g)$, and suppose that $\varphi: U \longrightarrow \mathbb{R}$ is a smooth function. The horizontal gradient of the function $\varphi$ is defined to be the vector field $\nabla_{H} \varphi$ such that $d_{q} \varphi(v)=g\left(\nabla_{H} \varphi(q), v\right)$ for every $v \in H_{q}, q \in U$. One easily makes sure that if $\nabla_{H} \varphi$ is null f.d. on $U$ and $\gamma:[a, b] \longrightarrow U$ is t.f.d. (nspc.f.d.), then the function $[a, b] \ni t \longrightarrow \varphi(\gamma(t))$ is decreasing (nonincreasing).

\subsection{Statement of the Results}

In papers $[5,7]$, we studied on the contact sub-Lorentzian structures on $\mathbb{R}^{3}$. On the other hand, in [8,9] (generalized) Martinet sub-Lorentzian structures of Hamiltonian type of order $k$ were studied, i.e., structures that, among other conditions imposed on them, are not contact on a hypersurface or, speaking in another way, structures whose Martinet surface is smooth. As a next step, it is reasonable to consider structures with the simplest non-smooth Martinet surface $S$, i.e., where $S$ is a union of transversely intersecting smooth hypersurfaces. In order to formulate necessary assumptions, let us introduce a notion of the hyperbolic angle on a sub-Lorentzian manifold $(M, H, g)$. Let $v_{1}, v_{2} \in H_{q}, q \in M$, be t.f.d. vectors. The hyperbolic angle between $v_{1}$ and $v_{2}$ is the number $\varangle\left(v_{1}, v_{2}\right) \geq 0$ defined by

$$
\cosh \varangle\left(v_{1}, v_{2}\right)=-\frac{g\left(v_{1}, v_{2}\right)}{\left\|v_{1}\right\|\left\|v_{2}\right\|},
$$

where $\|v\|=|g(v, v)|^{1 / 2}$; by the reverse Schwarz inequality $-\frac{g\left(v_{1}, v_{2}\right)}{\left\|v_{1}\right\|\left\|v_{2}\right\|} \geq 1$, so the definition makes sense. If $L_{1}=\operatorname{Span}\left\{v_{1}\right\}, L_{2}=\operatorname{Span}\left\{v_{2}\right\}$ are 1-dimensional timelike linear subspaces in $H_{q}$ with $v_{1}, v_{2}$ being chosen to be t.f.d., then we put

$$
\varangle\left(L_{1}, L_{2}\right)=\varangle\left(v_{1}, v_{2}\right)
$$

Now, we come to the precise definition of the type of sub-Lorentzian structures that we are going to consider in this paper. So let $H$ be a bracket generating distribution of constant rank equal to 2 , defined in a neighborhood $U$ of $0 \in \mathbb{R}^{3}$. We say that $H$ satisfies the condition $\left(M_{2,2}\right)$ if the following conditions are satisfied:

(i) There exist smooth hypersurfaces $S_{1}$ and $S_{2}$ such that the intersection $\Gamma=S_{1} \cap S_{2}$ is smooth of dimension 1, contains the origin, and is transverse to $H$; moreover, for each $q \in \Gamma, \operatorname{dim}\left(T_{q} S_{i} \cap H_{q}\right)=1, i=1,2$;

(ii) $\quad H$ defines a contact structure on $U \backslash\left(S_{1} \cup S_{2}\right)$;

(iii) $H_{q}^{2}=H_{q}$ and $H_{q}^{3}=T_{q} R^{3}$ whenever $q \in\left(S_{1} \cup S_{2}\right) \backslash \Gamma$;

(iv) $H_{q}^{4}=T_{q} R^{3}$ whenever $q \in \Gamma$.

The set $S=S_{1} \cup S_{2}$ will be called the Martinet surface for $H$. Note that $S$ is foliated by abnormal curves for the distribution $H$. Next, we chose a Lorentzian metric $g$ on $H$ in the way similar as in $[8,9]$ :

(v) The field of directions $S_{i} \ni q \longrightarrow T_{q} S_{i} \cap H_{q}$ is timelike, $i=1,2$;

(vi) The function $S_{1} \cap S_{2} \ni q \longrightarrow \varangle\left(T_{q} S_{1} \cap H_{q}, T_{q} S_{2} \cap H_{q}\right)$ is constant;

(vii) The abnormal curves foliating $S$ are, up to a change of parameter, t.f.d. Hamiltonian geodesics.

As we shall see, the two latter assumptions are used only in the process of constructing our normal form. 
We will say that a sub-Lorentzian structure $(H, g)$ is of type $M_{2,2}$ if it satisfies conditions (i)-(vii). The sub-Lorentzian structure $(H, g)$ is analytic if all objects entering its definition (e.g., the Martinet surface) are analytic.

Theorem 1.1 Let $(H, g)$ be a time-oriented analytic sub-Lorentzian structure of type $M_{2,2}$ defined on a neighborhood $U$ of the origin in $\mathbb{R}^{3}$. Then, provided that $U$ is sufficiently small, there exist analytic coordinates $x, y, z$ on $U$ in which $(H, g)$ has an orthonormal frame in the normal form

$$
\begin{aligned}
& X=\frac{\partial}{\partial x}+y \varphi\left(y \frac{\partial}{\partial x}+x \frac{\partial}{\partial y}\right)+\frac{1}{2} y\left(y-c_{1} x\right)\left(y-c_{2} x\right)(1+\psi) \frac{\partial}{\partial z} \\
& Y=\frac{\partial}{\partial y}-x \varphi\left(y \frac{\partial}{\partial x}+x \frac{\partial}{\partial y}\right)-\frac{1}{2} x\left(y-c_{1} x\right)\left(y-c_{2} x\right)(1+\psi) \frac{\partial}{\partial z}
\end{aligned}
$$

where $X$ is a time orientation; $c_{1}$ and $c_{2}$ are constants such that $-1<c_{2}<c_{1}<1$, $S=\left\{y=c_{1} x\right\} \cup\left\{y=c_{2} x\right\}$ is the Martinet surface for $H$; and $\varphi$ and $\psi$ are analytic functions on $U, \psi(0,0, z)=0$.

Using Theorem 1.1, we then investigate the structure of reachable sets. Let

$$
W=W\left(c_{1}, c_{2}\right)=c_{1} c_{2}+2 c_{1}-2 c_{2}-1 .
$$

It is not difficult to see what the geometric interpretation of Eq. 1.2 is. Let $\alpha=$ $\varangle\left(T_{q} S_{1} \cap H_{q}, T_{q} S_{2} \cap H_{q}\right)$ for a $q \in \Gamma$; Eq. 1.1 implies that $\cosh \alpha=\frac{1-c_{1} c_{2}}{\sqrt{1-c_{1}^{2}} \sqrt{1-c_{2}^{2}}}$ and at the same time $\sinh \alpha=\frac{c_{1}-c_{2}}{\sqrt{1-c_{1}^{2}} \sqrt{1-c_{2}^{2}}}$. Thus,

$$
\frac{W}{\sqrt{1-c_{1}^{2}} \sqrt{1-c_{2}^{2}}}=-\cosh \alpha+2 \sinh \alpha
$$

is an invariant for metrics of type $M_{2,2}$. In particular, the sign of $W$ has an invariant meaning and does not depend on the choice of coordinates. Note by the way that $W>0$ ( $W<0$, $W=0)$ if and only if $\tanh \alpha>\frac{1}{2}\left(\tanh \alpha<\frac{1}{2}, \tanh \alpha=\frac{1}{2}\right)$. As we are about to see, the sign of $W$ is determinative for the structure of reachable sets. More precisely, we will prove two theorems.

Theorem 1.2 Suppose that $(H, g)$ is a sub-Lorentzian structure defined on a suitably small normal neighborhood $U$ of the origin by an orthonormal frame $X, Y$ in the normal form (1.1) with $X$ being a time orientation. Then, if $W\left(c_{1}, c_{2}\right)>0$, there exist two analytic functions $\eta_{1}, \eta_{2}: U \longrightarrow \mathbb{R}$ such that the reachable sets from the origin for $(H, g)$ are of the following form:

$$
\begin{gathered}
J^{+}(0, U)=N^{+}(0, U)=A_{1} \cup A_{2}, \\
I^{+}(0, U)=\operatorname{int}\left(A_{1} \cup A_{2}\right),
\end{gathered}
$$

where

$$
\begin{aligned}
& A_{1}=\left\{(x, y, z) \in U: \eta_{1}(x, y, z) \leq 0\right\} \cap\{x \geq 0, z \geq 0\}, \\
& A_{2}=\left\{(x, y, z) \in U: \eta_{2}(x, y, z) \leq 0\right\} \cap\{x \geq 0, z \leq 0\} .
\end{aligned}
$$

In particular, the three reachable sets are semi-analytic. 
Note that in cases covered by Theorem 1.2, there are no timelike curves in the boundary $\tilde{\partial} J^{+}(0, U)$, and the structure of reachable sets is the same as in the contact case.

Theorem 1.3 Suppose that $(H, g)$ is a sub-Lorentzian structure defined on a suitably small normal neighborhood $U$ of the origin by an orthonormal frame $X, Y$ in the normal form (1.1) with $X$ being a time orientation. Then, if $W\left(c_{1}, c_{2}\right)<0$, there exist six analytic functions $\eta_{1}, \eta_{2}, \xi_{i j}: U \longrightarrow \mathbb{R}, i, j=1,2$, and two 2-dimensional semi-analytic sets $\Sigma_{1}, \Sigma_{2}$ with the property that $U \cap\{x \geq 0\} \cap\{z \geq 0\} \cap\left\{c_{2} x \leq y \leq x\right\} \backslash \Sigma_{1}$ has two connected components $\Sigma_{1}^{+}, \Sigma_{1}^{-}$, and $U \cap\{x \geq 0\} \cap\{z \leq 0\} \cap\left\{-x \leq y \leq c_{1} x\right\} \backslash \Sigma_{2}$ has two connected components $\Sigma_{2}^{+}, \Sigma_{2}^{-}$, such that

$$
\begin{gathered}
J^{+}(0, U)=A_{1} \cup \ldots \cup A_{6}, \\
I^{+}(0, U)=\operatorname{int}\left(A_{1} \cup \ldots \cup A_{6}\right) \cup A_{7} \cup A_{8}, \\
N^{+}(0, U)=\operatorname{int}\left(A_{1} \cup \ldots \cup A_{6}\right) \cup\left(\left\{\eta_{1}=0\right\} \cap \Sigma_{1}^{+}\right) \cup\left(\left\{\eta_{2}=0\right\} \cap \Sigma_{2}^{-}\right)
\end{gathered}
$$

where

$$
\begin{aligned}
& A_{1}=\left\{(x, y, z) \in U: \eta_{1}(x, y, z) \leq 0\right\} \cap \Sigma_{1}^{+}, \\
& A_{2}=\left\{(x, y, z) \in U: \xi_{11}(x, y, z) \leq 0\right\} \cap \Sigma_{1}^{-}, \\
& A_{3}=\left\{(x, y, z) \in U: \xi_{12}(x, y, z) \leq 0\right\} \cap\left\{-x \leq y \leq c_{2} x\right\} \cap\{z \geq 0\}, \\
& A_{4}=\left\{(x, y, z) \in U: \eta_{2}(x, y, z) \leq 0\right\} \cap \Sigma_{2}^{-}, \\
& A_{5}=\left\{(x, y, z) \in U: \xi_{21}(x, y, z) \leq 0\right\} \cap \Sigma_{2}^{+}, \\
& A_{6}=\left\{(x, y, z) \in U: \xi_{22}(x, y, z) \leq 0\right\} \cap\left\{c_{1} x \leq y \leq x\right\} \cap\{z \leq 0\}, \\
& A_{7}=\left\{(x, y, z) \in U: y=c_{1} x, x \geq 0, z=0\right\}, \\
& A_{8}=\left\{(x, y, z) \in U: y=c_{2} x, x \geq 0, z=0\right\} .
\end{aligned}
$$

In particular, the three reachable sets are semi-analytic.

Note that in cases covered by Theorem 1.3, there are two timelike curves on the boundary $\tilde{\partial} J^{+}(0, U)$. It is also seen that in such cases, neither $I^{+}(0, U)$ is open nor $N^{+}(0, U)$ is closed.

The structure of all geometrically optimal curves in cases treated in Theorems 1.2 and 1.3 is described in Section 2. Let us notice that proofs of Theorems 1.2 and 1.3 give a sort of "algorithm" for computing the reachable sets. Moreover, the presented results bear a geometric character, so having proved Theorems 1.2 and 1.3 and applying a remark similar to Remark 4.4 in [9], we no longer have to transform our structure to the normal form in order to compute reachable sets.

Similarly as it was done in some previous papers by the author, all the above results can be applied to the study on reachable sets for control affine systems induced by subLorentzian metrics of type $M_{2,2}$. Let

$$
\dot{q}=X+u Y, \quad u \in[a, b],
$$

be a control affine system defined on $M$, where $M$ is an open subset of $\mathbb{R}^{3}$ (or a 3-manifold). We suppose the fields $X, Y$ to be linearly independent. Fix a point $q_{0} \in M$ and its neighborhood $U \subset M$. We will consider the reachable set $\mathcal{A}_{[a, b]}\left(q_{0}, U\right)$ from $q_{0}$ for the system 
(1.3) which is defined to be the set of endpoints of all trajectories of Eq. 1.3 that are contained in $U$, initiate at $q_{0}$, and are generated by measurable controls $u:[0, T] \longrightarrow[a, b]$; here, $T=T(u)$ depends on a control. It was proved in [9], Lemma 1.2, that the reachable set $\mathcal{A}_{[a, b]}\left(q_{0}, U\right)$ is equal to the future nonspacelike reachable set for the time-oriented sub-Lorentzian structure $\left(H, g^{a, b}\right)$ defined on $M$ by declaring the frame

$$
\begin{aligned}
& Z^{a, b}=X+\frac{1}{2}(b+a) Y \\
& W^{a, b}=\frac{1}{2}(b-a) Y
\end{aligned}
$$

to be orthonormal with a time orientation $Z^{a, b}$ (in [9], we studied also on reachable sets $\mathcal{A}_{(a, b)}\left(q_{0}, U\right)$ and $\left.\mathcal{A}_{\{a, b\}}\left(q_{0}, U\right)\right)$. Let us recall a notion of singular trajectories (cf. [4]) for affine control systems. So, a trajectory $\gamma:[0, T] \longrightarrow U$ of Eq. 1.3 is called a singular trajectory if it is generated by a control $u(t)$ with values in the open interval $(a, b)$ and is an abnormal curve for the distribution $H$. It is worth noting that singular trajectories satisfy necessary conditions for geometric optimality of Pontriagin maximum principle. In terms of the sub-Lorentzian metric $\left(H, g^{a, b}\right)$, singular trajectories are exactly timelike abnormal curves.

Now, suppose that $X, Y$ is an orthonormal frame for a metric $(H, g)$ of type $M_{2,2}$. We say that the system $\dot{q}=X+u Y, u \in[a, b]$, is typical in class $M_{2,2}$ if $W\left(\frac{2 c_{1}-b-a}{b-a}, \frac{2 c_{2}-b-a}{b-a}\right) \neq$ 0 (cf. 5.9), where $c_{1}$ and $c_{2}$ are constants obtained by transforming $(H, g)$ to normal form (1.1). Then, we can prove the following:

Theorem 1.4 Suppose that the system (1.3) is typical in class $M_{2,2}$. If it has $k$ geometrically optimal singular trajectories initiating at $q_{0}$ (in our case, $k=0,1,2$ ), then the minimal number of analytic functions that one needs to describe the reachable set $\mathcal{A}_{[a, b]}\left(q_{0}, U\right)$ is $2+2 k$.

The above theorem also holds for all cases treated in [9]. Thus, the presence on the boundary $\tilde{\partial} \mathcal{A}_{[a, b]}\left(q_{0}, U\right)$ of a singular trajectory initiating at $q_{0}$ increases (at least in the described cases) by two the minimal number of analytic functions needed for describing the reachable set $\mathcal{A}_{[a, b]}\left(q_{0}, U\right)$. It would be interesting to know whether this observation can be extended to a more general class of (not necessarily affine) control systems.

\subsection{Organizations of the Paper}

Section 2 is devoted to computing reachable sets for the so-called flat structures-they correspond to normal form (1.1) with $\varphi$ and $\psi$ set to be equal to zero. In Section 3, we compute normal forms. More precisely, we prove Theorem 3.1 which gives normal forms in a more general situation than that treated in the present paper and which can be a starting point for further studies. Theorem 1.1 is then a corollary of Theorem 3.1. In Section 4, we generalize global results from Section 2 to local results in a general (i.e., nonflat) situation of type $M_{2,2}$ in cases where $W\left(c_{1}, c_{2}\right) \neq 0$. In Section 5, we apply the results obtained for sub-Lorentzian structures to control affine systems. Proofs of the lemmas from Section 2.2 are contained in Section 6. Section 7 contains 3-dimensional visualizations of examples of reachable sets studied in Section 2. In the Appendix, we state some corollaries concerning the image under exponential mapping and also conjugate and cut loci.

Some proofs of the results presented in the paper are omitted since they are similar to those from [9]. 


\section{Reachable Sets in the Flat Case}

In this section, we study on reachable sets from the origin for the sub-Lorentzian structure $(\hat{H}, \hat{g})$ defined by an orthonormal basis

$$
\begin{aligned}
& \hat{X}=\frac{\partial}{\partial x}+\frac{1}{2} y\left(y-c_{1} x\right)\left(y-c_{2} x\right) \frac{\partial}{\partial z} \\
& \hat{Y}=\frac{\partial}{\partial y}-\frac{1}{2} x\left(y-c_{1} x\right)\left(y-c_{2} x\right) \frac{\partial}{\partial z}
\end{aligned}
$$

with a time orientation $\hat{X}$ where we assume that $-1<c_{2}<c_{1}<1$. Let $S_{i}=\{(x, y, z)$ : $\left.y=c_{i} x\right\}$. We see that the Martinet surface $S$ in our case is equal to $S_{1} \cup S_{2}$. The structure (or a metric) $(\hat{H}, \hat{g})$ will be called flat. This is because Eq. 2.1 is a particular case of Eq. 1.1 where $\varphi$ and $\psi$ has been set to zero. Hence, any structure as in Eq. 1.1 can be regarded as a perturbation of the flat structure; see Section 4 for some applications of this observation.

Reachable sets from the origin for Eq. 2.1 will be denoted respectively by $\hat{J}^{+}(0)=$ $J^{+}\left(0, \mathbb{R}^{3}\right), \hat{I}^{+}(0)=I^{+}\left(0, \mathbb{R}^{3}\right)$, and $\hat{N}^{+}(0)=N^{+}\left(0, \mathbb{R}^{3}\right)$. First of all, it is obvious that

$$
\hat{I}^{+}(0) \subset\{-x<y<x, x>0\}
$$

and

$$
\hat{J}^{+}(0) \subset\{-x \leq y \leq x, x \geq 0\} .
$$

As in the previous papers by the author, the key role in the process of constructing functions describing reachable sets is played by the signs of the $z$-coordinates of the fields

$$
\begin{aligned}
& \hat{X}-\hat{Y}=\frac{\partial}{\partial x}-\frac{\partial}{\partial y}+\frac{1}{2}(y+x)\left(y-c_{1} x\right)\left(y-c_{2} x\right) \frac{\partial}{\partial z} \\
& \hat{X}+\hat{Y}=\frac{\partial}{\partial x}+\frac{\partial}{\partial y}+\frac{1}{2}(y-x)\left(y-c_{1} x\right)\left(y-c_{2} x\right) \frac{\partial}{\partial z} .
\end{aligned}
$$

Let

$$
\Gamma_{1}=\{(x, x, z): x, z \in \mathbb{R}\}
$$

and

$$
\Gamma_{2}=\{(x,-x, z): x, z \in \mathbb{R}\} .
$$

Since $(\hat{X}-\hat{Y})(z)>0$ on $\Gamma_{1}$ and $(\hat{X}+\hat{Y})(z)<0$ on $\Gamma_{2}$, similarly as in [7-9], we construct two functions $\hat{\eta}_{1}$ and $\hat{\eta}_{2} . \hat{\eta}_{1}$ is the solution to the Cauchy problem

$$
(\hat{X}-\hat{Y})(\eta)=0,\left.\quad \eta\right|_{\Gamma_{1}}(x, x, z)=z,
$$

and $\hat{\eta}_{2}$ is the solution to the Cauchy problem

$$
(\hat{X}+\hat{Y})(\eta)=0,\left.\quad \eta\right|_{\Gamma_{2}}(x,-x, z)=-z
$$

After calculations, we obtain

$$
\begin{gathered}
\hat{\eta}_{1}(x, y, z)=z-\frac{x^{2}-y^{2}}{48}\left(\left(7 c_{1} c_{2}-2 c_{1}-2 c_{2}+1\right) x^{2}+4\left(c_{1} c_{2}-2 c_{1}-2 c_{2}+1\right) x y\right. \\
\left.+\left(c_{1} c_{2}-2 c_{2}-2 c_{1}+7\right) y^{2}\right)
\end{gathered}
$$

and

$$
\begin{aligned}
\hat{\eta}_{2}(x, y, z)=-z-\frac{x^{2}-y^{2}}{48} & \left(\left(7 c_{1} c_{2}+2 c_{1}+2 c_{2}+1\right) x^{2}-4\left(c_{1} c_{2}+2 c_{1}+2 c_{2}+1\right) x y\right. \\
& \left.+\left(c_{1} c_{2}+2 c_{2}+2 c_{1}+7\right) y^{2}\right) .
\end{aligned}
$$


As in the previous papers, we need to know the horizontal gradient $\nabla_{\hat{H}} \hat{\eta}_{i}$ of the function $\hat{\eta}_{i}$ with respect to $(\hat{H}, \hat{g}), i=1,2$. Clearly, $\nabla_{\hat{H}} \hat{\eta}_{1}=-\hat{X}\left(\hat{\eta}_{1}\right)(\hat{X}-\hat{Y})$ where

$$
\begin{gathered}
\hat{X}\left(\hat{\eta}_{1}\right)=-\frac{1}{12}(x-y)\left(\left(7 c_{1} c_{2}-2 c_{1}-2 c_{2}+1\right) x^{2}+4\left(c_{1} c_{2}-2 c_{1}-2 c_{2}+1\right) x y\right. \\
\left.+\left(c_{1} c_{2}-2 c_{1}-2 c_{2}+7\right) y^{2}\right)
\end{gathered}
$$

and $\nabla_{\hat{H}} \hat{\eta}_{2}=-\hat{X}\left(\hat{\eta}_{2}\right)(\hat{X}+\hat{Y})$ where

$$
\begin{gathered}
\hat{X}\left(\hat{\eta}_{2}\right)=-\frac{1}{12}(x+y)\left(\left(7 c_{1} c_{2}+2 c_{1}+2 c_{2}+1\right) x^{2}-4\left(c_{1} c_{2}+2 c_{1}+2 c_{2}+1\right) x y\right. \\
\left.+\left(c_{1} c_{2}+2 c_{1}+2 c_{2}+7\right) y^{2}\right) .
\end{gathered}
$$

Recall that in (1.2) in Section 1, we defined the polynomial $W=c_{1} c_{2}+2 c_{1}-2 c_{2}-1$. As it was announced, the sign of $W$ determines the structure of the reachable set for Eq. 2.1. Indeed, it is easy to check that

$$
\hat{X}\left(\hat{\eta}_{1}\right)=-\frac{1}{12}(x-y)\left(c_{1} c_{2}-2 c_{1}-2 c_{2}+7\right)\left(y-E_{1} x\right)\left(y-E_{2} x\right)
$$

with

$$
\begin{aligned}
& E_{1}=\frac{1}{c_{1} c_{2}-2 c_{1}-2 c_{2}+7}\left(-2 c_{1} c_{2}+4 c_{1}+4 c_{2}-2+\sqrt{-3\left(c_{1} c_{2}-2 c_{1}+2 c_{2}-1\right) W}\right) \\
& E_{2}=\frac{1}{c_{1} c_{2}-2 c_{1}-2 c_{2}+7}\left(-2 c_{1} c_{2}+4 c_{1}+4 c_{2}-2-\sqrt{-3\left(c_{1} c_{2}-2 c_{1}+2 c_{2}-1\right) W}\right)
\end{aligned}
$$

and

$$
\hat{X}\left(\hat{\eta}_{2}\right)=-\frac{1}{12}(x+y)\left(c_{1} c_{2}+2 c_{1}+2 c_{2}+7\right)\left(y-E_{3} x\right)\left(y-E_{4} x\right)
$$

with

$$
\begin{aligned}
& E_{3}=\frac{1}{c_{1} c_{2}+2 c_{1}+2 c_{2}+7}\left(2 c_{1} c_{2}+4 c_{1}+4 c_{2}+2+\sqrt{-3\left(c_{1} c_{2}-2 c_{1}+2 c_{2}-1\right) W}\right) \\
& E_{4}=\frac{1}{c_{1} c_{2}+2 c_{1}+2 c_{2}+7}\left(2 c_{1} c_{2}+4 c_{1}+4 c_{2}+2-\sqrt{-3\left(c_{1} c_{2}-2 c_{1}+2 c_{2}-1\right) W}\right)
\end{aligned} .
$$

Let us notice here that $c_{1} c_{2}-2 c_{1}+2 c_{2}-1=\left(c_{1} c_{2}-1\right)-2\left(c_{1}-c_{2}\right)<0$ for all $c_{1}$ and $c_{2}$ such that $-1<c_{2}<c_{1}<1$.

2.1 The Case $W<0$

This case is the simplest because, as it can be seen from Eqs. 2.6 and 2.8, $\nabla_{\hat{H}} \hat{\eta}_{i}, i=1,2$ is null f.d. in the whole sector $\{-x<y<x\}$. Thus, using similar arguments as, e.g., in [7] or [8], we have

Proposition 2.1 If $W<0$ then

$$
\hat{J}^{+}(0)=\hat{N}^{+}(0)=\hat{A}_{1} \cup \hat{A}_{2}
$$

and

$$
\hat{I}^{+}(0)=\operatorname{int}\left(\hat{A}_{1} \cup \hat{A}_{2}\right),
$$

where

$$
\begin{aligned}
& \hat{A}_{1}=\left\{(x, y, z) \in \mathbb{R}^{3}: \hat{\eta}_{1}(x, y, z) \leq 0\right\} \cap\{x \geq 0, z \geq 0\}, \\
& \hat{A}_{2}=\left\{(x, y, z) \in \mathbb{R}^{3}: \hat{\eta}_{2}(x, y, z) \leq 0\right\} \cap\{x \geq 0, z \leq 0\} .
\end{aligned}
$$


Let us remark that

$$
\begin{aligned}
& \hat{\eta}_{1}\left(x, c_{1} x, 0\right)=\frac{1}{48} x^{4}\left(c_{1}-1\right)^{2}\left(c_{1}+1\right)^{2}\left(c_{1} c_{2}-2 c_{1}+2 c_{2}-1\right)<0, \\
& \hat{\eta}_{1}\left(x, c_{2} x, 0\right)=\frac{1}{48} x^{4}\left(c_{2}-1\right)^{2}\left(c_{2}+1\right)^{2} W<0, \\
& \hat{\eta}_{2}\left(x, c_{1} x, 0\right)=\frac{1}{48} x^{4}\left(c_{1}-1\right)^{2}\left(c_{1}+1\right)^{2} W<0, \text { and } \\
& \hat{\eta}_{2}\left(x, c_{2} x, 0\right)=\frac{1}{48} x^{4}\left(c_{2}-1\right)^{2}\left(c_{2}+1\right)^{2}\left(c_{1} c_{2}-2 c_{1}+2 c_{2}-1\right)<0 .
\end{aligned}
$$

This means that there are no geometrically optimal timelike (and hence abnormal) curves starting from 0 , and in what follows, only two functions suffice to describe the reachable sets.

To visualize better how the reachable set looks like, let us list all geometrically optimal curves. To this end, introduce the following notation. If $Z_{1}, Z_{2}$ are two vector fields on $\mathbb{R}^{3}$, then by $Z_{1} Z_{2}$, we will mean the curve which is a concatenation of a segment of the trajectory of $Z_{1}$ starting from 0 , and a segment of a trajectory of $Z_{2}$. Using such a notation, every geometrically optimal curve is either $(\hat{X}+\hat{Y})(\hat{X}-\hat{Y})$ or $(\hat{X}-\hat{Y})(\hat{X}+\hat{Y})$. The intersection of $\partial \hat{J}^{+}(0)$ with the plane $\{x=$ const $>0\}$ is schematically presented in Fig. 1.

Points $A$ and $B$ lie on the plane $\{z=0\} . A$ (resp. $B$ ) corresponds to half-line $\{y=-x, z=0\}$ (resp. $\{y=-x, z=0\}$ ). The curve $B A$ located above the straight line joining $A$ and $B$ represents $\partial \hat{J}^{+}(0) \cap\{z \geq 0\}$ and is formed by the trajectories $(\hat{X}+\hat{Y})$ $(\hat{X}-\hat{Y})$; the curve $A B$ located below this straight line represents $\partial \hat{J}^{+}(0) \cap\{z \leq 0\}$ and is formed by the trajectories $(\hat{X}-\hat{Y})(\hat{X}+\hat{Y})$.

\subsection{The Case $W>0$}

In order to simplify reading of this subsection, proofs of all lemmas are moved to Section 6.

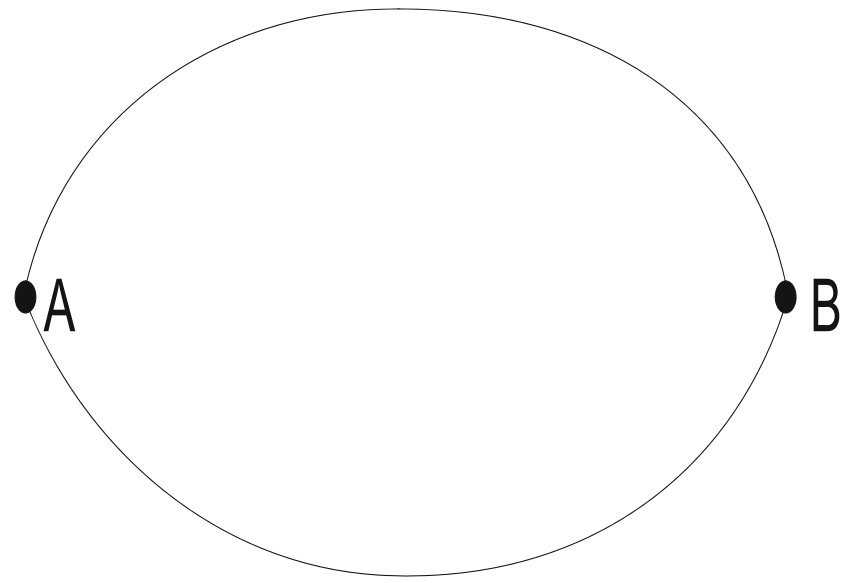

Fig. 1 The set $\partial \hat{J}^{+}(0) \cap\{x=$ const $>0\}$ in the case $W<0$ 
This case is more complicated since now $E_{i}, i=1, \ldots, 4$ are real. First of all, we must examine constants $E_{1}, \ldots, E_{4}$. Obviously, $E_{2}<E_{1}$ and $E_{4}<E_{3}$. Moreover, we have two lemmas.

Lemma 2.1 The following inequalities hold true: $-1<E_{2}<E_{1}<1, E_{i}<c_{i}, i=1,2$.

Lemma 2.2 The following inequalities hold true: $-1<E_{4}<E_{3}<1, c_{2}<E_{4}, c_{1}<E_{3}$.

Using Eqs. 2.6 and 2.8, we conclude that $\nabla_{\hat{H}} \hat{\eta}_{1}$ is null f.d. on $\left\{E_{1} x<y<x\right\}$, while $\nabla_{\hat{H}} \hat{\eta}_{2}$ is null f.d. on $\left\{-x<y<E_{4} x\right\}$. Hence, we need more functions to describe the reachable sets from the origin.

First, we will compute $\hat{J}^{+}(0) \cap\{z \geq 0\}$. Since $(\hat{X}+\hat{Y})(z)>0$ for $y>c_{2} x$, and $(\hat{X}-\hat{Y})(z)>0$ for $y<c_{2} x$, everything in a neighborhood of the plane $\left\{y=c_{2} x\right\}$, it is natural to consider the following Cauchy problems:

$$
(\hat{X}+\hat{Y})(\eta)=0, \eta\left(x, c_{2} x, z\right)=z
$$

with the solution equal to

$$
\hat{\xi}_{11}(x, y, z)=z-\frac{1}{12} \frac{(x-y)\left(y-c_{2} x\right)^{2}}{\left(1-c_{2}\right)^{2}}\left(\left(-2 c_{1} c_{2}+3 c_{1}-c_{2}\right) x+\left(-c_{1}+3 c_{2}-2\right) y\right) \text {, }
$$

and

$$
(\hat{X}-\hat{Y})(\eta)=0, \quad \eta\left(x, c_{2} x, z\right)=z
$$

with the solution equal to

$$
\hat{\xi}_{12}(x, y, z)=z-\frac{1}{12} \frac{(x+y)\left(c_{2} x-y\right)^{2}}{\left(c_{2}+1\right)^{2}}\left(\left(2 c_{1} c_{2}+3 c_{1}-c_{2}\right) x+\left(c_{1}-3 c_{2}-2\right) y\right) .
$$

Now, we examine horizontal gradients $\nabla_{\hat{H}} \hat{\xi}_{1 i}, i=1,2$. So $\nabla_{\hat{H}} \hat{\xi}_{11}=-\hat{X}\left(\hat{\xi}_{11}\right)(\hat{X}+\hat{Y})$ with

$$
\hat{X}\left(\hat{\xi}_{11}\right)=\frac{\left(y-c_{2} x\right)^{2}}{3\left(c_{2}-1\right)^{2}}\left(\left(2 c_{1} c_{2}-3 c_{1}+c_{2}\right) x+\left(c_{1}-3 c_{2}+2\right) y\right)
$$

and $\nabla_{\hat{H}} \hat{\xi}_{12}=-\hat{X}\left(\hat{\xi}_{12}\right)(\hat{X}-\hat{Y})$ with

$$
\hat{X}\left(\hat{\xi}_{12}\right)=-\frac{\left(y-c_{2} x\right)^{2}}{3\left(c_{2}+1\right)^{2}}\left(\left(2 c_{1} c_{2}+3 c_{1}-c_{2}\right) x+\left(c_{1}-3 c_{2}-2\right) y\right) .
$$

Using Eq. 2.11, it is easy to see that $\nabla_{\hat{H}} \hat{\xi}_{12}$ is null f.d. in $\left\{-x<y<c_{2} x\right\}$. Indeed, if $c_{1}-3 c_{2}-2 \geq 0$, then we have $\left(2 c_{1} c_{2}+3 c_{1}-c_{2}\right) x+\left(c_{1}-3 c_{2}-2\right) y \geq$ $2\left(c_{2}+1\right)\left(c_{1}+1\right) x>0$. If, on the other hand, $c_{1}-3 c_{2}-2<0$, then $\left(2 c_{1} c_{2}+3 c_{1}-c_{2}\right) x+\left(c_{1}-3 c_{2}-2\right) y>\left(2 c_{1} c_{2}+3 c_{1}-c_{2}\right) x+c_{2}\left(c_{1}-3 c_{2}-2\right) x=$ $3\left(c_{2}+1\right)\left(c_{1}-c_{2}\right) x>0$. Also, since $c_{1}-3 c_{2}+2=c_{1}-c_{2}+2\left(1-c_{2}\right)>0$, we see that $\nabla_{\hat{H}} \hat{\xi}_{11}$ is null f.d. for $y<-\frac{2 c_{1} c_{2}-3 c_{1}+c_{2}}{c_{1}-3 c_{2}+2} x$.

Lemma $2.3 c_{1}<-\frac{2 c_{1} c_{2}-3 c_{1}+c_{2}}{c_{1}-3 c_{2}+2}<1$.

Now, by Lemmas 2.1 and 2.3, it is clear that $E_{1}<-\frac{2 c_{1} c_{2}+c_{2}-3 c_{1}}{c_{1}+2-3 c_{2}}$. We will compute the intersection $\left\{\hat{\eta}_{1}=0\right\} \cap\left\{\hat{\xi}_{11}=0\right\} \cap\left\{E_{1} x<y<-\frac{2 c_{1} c_{2}+c_{2}-3 c_{1}}{c_{1}+2-3 c_{2}} x\right\}$. Evidently,

$$
\hat{\xi}_{11}\left(x, c_{2} x, z\right)-\hat{\eta}_{1}\left(x, c_{2} x, z\right)=-\frac{1}{48} x^{4}\left(c_{2}-1\right)^{2}\left(c_{2}+1\right)^{2} W<0 .
$$


On the other hand,

$$
\begin{aligned}
& \hat{\xi}_{11}\left(x, c_{1} x, z\right)-\hat{\eta}_{1}\left(x, c_{1} x, z\right)=-\frac{1}{48} \frac{\left(c_{1}-1\right)^{2}}{\left(c_{2}-1\right)^{2}} x^{4} \\
& \left(4\left(c_{1}-c_{2}\right)^{3}+\left(c_{2}-1\right)^{2}\left(c_{1}+1\right)^{2}\left(c_{1} c_{2}-2 c_{1}+2 c_{2}-1\right)\right) .
\end{aligned}
$$

We need the following:

Lemma 2.4 Let $f(x, y)=4(x-y)^{3}+(y-1)^{2}(x+1)^{2}(x y-2 x+2 y-1)$ be a function considered on the set $D=\{(x, y):-1<y<x<1\}$. Then $f<0$ on $D$.

Lemma 2.4 and Eq. 2.13 give

$$
\hat{\xi}_{11}\left(x, c_{1} x, z\right)-\hat{\eta}_{1}\left(x, c_{1} x, z\right)>0 .
$$

Moreover, $(X+Y)\left(\hat{\xi}_{11}-\hat{\eta}_{1}\right)=-(X+Y)\left(\hat{\eta}_{1}\right)<0$ on $\left\{c_{2} x<y<E_{1} x\right\}$, and $(X+Y)$ $\left(\hat{\xi}_{11}-\hat{\eta}_{1}\right)>0$ on $\left\{E_{1} x<y<x\right\}$. Now, let us sum up what we already know. By Eq. 2.12, the expression $\hat{\xi}_{1}-\hat{\eta}_{1}$ (which is a homogeneous polynomial in $x, y$ ) is negative on $y=c_{2} x$ and decreases along the trajectories of $X+Y$ in $\left\{c_{2} x<y<E_{1} x\right\}$. Then, $\hat{\xi}_{11}-\hat{\eta}_{1}$ starts to increase and for $y=c_{1} x$, it attains a positive value by Eq. 2.14. It follows that $\left\{\hat{\eta}_{1}=0\right\} \cap$ $\left\{\hat{\xi}_{11}=0\right\} \cap\left\{E_{1} x<y<-\frac{2 c_{1} c_{2}+c_{2}-3 c_{1}}{c_{1}+2-3 c_{2}} x\right\}$ is of the form $\left\{y=S_{1} x\right\}$ with $E_{1}<S_{1}<c_{1}$. In this way, we arrive at the following:

$$
\hat{J}^{+}(0) \cap\{z \geq 0\}=\hat{A}_{1} \cup \hat{A}_{2} \cup \hat{A}_{3}
$$

where

$$
\begin{gathered}
\hat{A}_{1}=\left\{\hat{\eta}_{1} \leq 0\right\} \cap\left\{S_{1} x \leq y \leq x\right\} \cap\{z \geq 0\}, \\
\hat{A}_{2}=\left\{\hat{\xi}_{11} \leq 0\right\} \cap\left\{c_{2} x \leq y \leq S_{1} x\right\} \cap\{z \geq 0\}, \\
\hat{A}_{3}=\left\{\hat{\xi}_{12} \leq 0\right\} \cap\left\{-x \leq y \leq c_{2} x\right\} \cap\{z \geq 0\} .
\end{gathered}
$$

The signs of $z$-coordinates of the fields $\hat{X}+\hat{Y}, \hat{X}-\hat{Y}$ needed in the computation of $\partial \hat{J}^{+}(0) \cap\{z>0\}$ are schematically illustrated in Fig. 2. Arrows pointing up correspond to the field $\hat{X}+\hat{Y}$, while those pointing down correspond to $\hat{X}-\hat{Y}$.

Now, we examine $\hat{J}^{+}(0) \cap\{z \leq 0\}$. Let us consider two Cauchy problems:

$$
(\hat{X}-\hat{Y})(\eta)=0, \eta\left(x, c_{1} x, z\right)=-z
$$

with the solution equal to

$$
\hat{\xi}_{21}(x, y, z)=-z-\frac{1}{12} \frac{(x+y)\left(c_{1} x-y\right)^{2}}{\left(1+c_{1}\right)^{2}}\left(\left(c_{1}-2 c_{1} c_{2}-3 c_{2}\right) x+\left(3 c_{1}-c_{2}+2\right) y\right),
$$

and

$$
(\hat{X}+\hat{Y})(\eta)=0, \eta\left(x, c_{1} x, z\right)=-z
$$

with the solution equal to

$$
\hat{\xi}_{22}(x, y, z)=-z+\frac{1}{12} \frac{(x-y)\left(y-c_{1} x\right)^{2}}{\left(1-c_{1}\right)^{2}}\left(\left(-2 c_{1} c_{2}-c_{1}+3 c_{2}\right) x+\left(3 c_{1}-c_{2}-2\right) y\right) .
$$




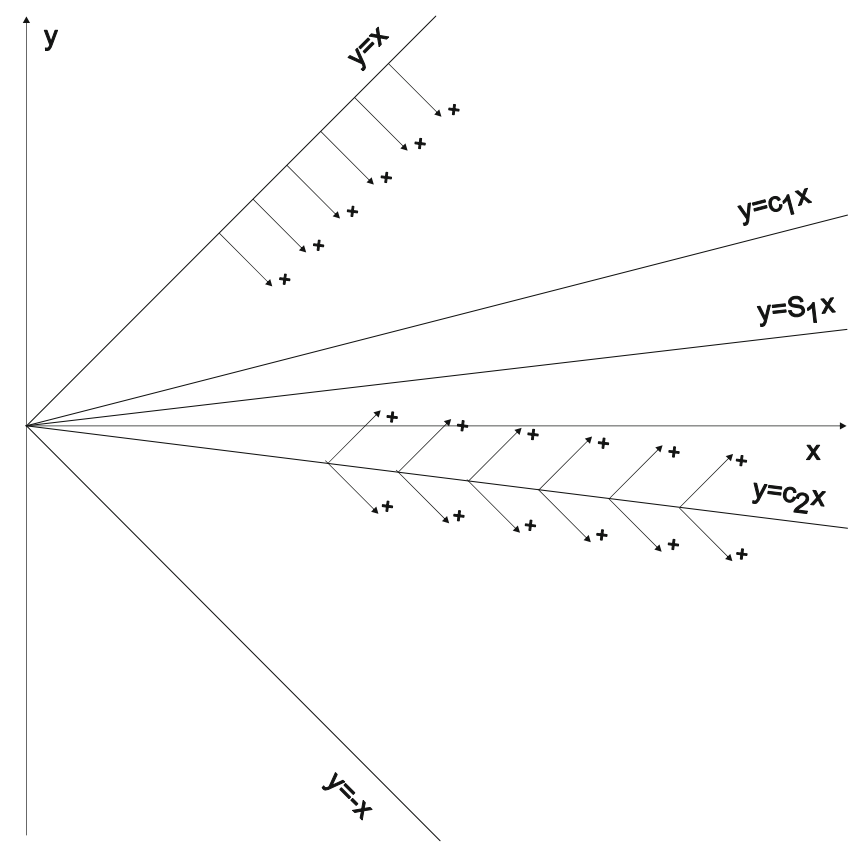

Fig. 2 The case $W>0$. Lines on the plane $\{z=0\}$ along which the fields $\hat{X}+\hat{Y}, \hat{X}-\hat{Y}$ point toward the positive direction of the $z$-axis

As above, we need to know the regions where horizontal gradients $\nabla_{\hat{H}} \hat{\xi}_{2 i}, i=1,2$ are suitably directed. After calculations,

$$
\hat{X}\left(\hat{\xi}_{21}\right)=\frac{\left(c_{1} x-y\right)^{2}}{3\left(c_{1}+1\right)^{2}}\left(\left(2 c_{1} c_{2}-c_{1}+3 c_{2}\right) x+\left(-3 c_{1}+c_{2}-2\right) y\right)
$$

and

$$
\hat{X}\left(\hat{\xi}_{22}\right)=\frac{\left(y-c_{1} x\right)^{2}}{3\left(1-c_{1}\right)^{2}}\left(\left(-2 c_{1} c_{2}-c_{1}+3 c_{2}\right) x+\left(3 c_{1}-c_{2}-2\right) y\right) .
$$

Equation 2.19 immediately yields that $\nabla_{\hat{H}} \hat{\xi}_{22}$ is null f.d. in $\left\{c_{1} x<y<x\right\}$. Indeed, $\left(-2 c_{1} c_{2}-c_{1}+3 c_{2}\right) x+\left(3 c_{1}-c_{2}-2\right) y<-2\left(c_{2}-1\right)\left(c_{1}-1\right) x<0$ in this sector whenever $3 c_{1}-c_{2}-2 \geq 0$. On the other hand, if $3 c_{1}-c_{2}-2<0$, then $\left(-2 c_{1} c_{2}-c_{1}+3 c_{2}\right) x+\left(3 c_{1}-c_{2}-2\right) y<\left(-2 c_{1} c_{2}-c_{1}+3 c_{2}\right) x+c_{1}\left(3 c_{1}-c_{2}-2\right) x=$ $3\left(c_{1}-1\right)\left(c_{1}-c_{2}\right) x<0$. Also by Eq. 2.18, we know that $\nabla_{H} \hat{\xi}_{21}$ is null f.d. for $y>$ $-\frac{2 c_{1} c_{2}-c_{1}+3 c_{2}}{-3 c_{1}+c_{2}-2} x$. Indeed, it is enough to notice that $-3 c_{1}+c_{2}-2=-\left(c_{1}-c_{2}\right)-$ $2\left(c_{1}+1\right)<0$.

Lemma $2.5-1<-\frac{2 c_{1} c_{2}-c_{1}+3 c_{2}}{-3 c_{1}+c_{2}-2}<c_{2}$.

Now, it follows that $-\frac{2 c_{1} c_{2}-c_{1}+3 c_{2}}{-3 c_{1}+c_{2}-2}<E_{4}$, and we will compute the intersection $\left\{\hat{\eta}_{2}=0\right\} \cap\left\{\hat{\xi}_{21}=0\right\} \cap\left\{-\frac{2 c_{1} c_{2}-c_{1}+3 c_{2}}{-3 c_{1}+c_{2}-2} x<y<E_{4} x\right\}$. We proceed similarly as above. So, first of all,

$$
\hat{\xi}_{21}\left(x, c_{1} x, z\right)-\hat{\eta}_{2}\left(x, c_{1} x, z\right)=-\frac{1}{48} x^{4}\left(c_{1}-1\right)^{2}\left(c_{1}+1\right)^{2} W<0 .
$$


Next,

$$
\begin{aligned}
& \hat{\xi}_{21}\left(x, c_{2} x, z\right)-\hat{\eta}_{2}\left(x, c_{2} x, z\right)=\frac{\left(c_{2}+1\right)^{2}}{48\left(c_{1}+1\right)^{2}} x^{4} \\
& \left(4\left(-c_{1}+c_{2}\right)^{3}-\left(c_{1}+1\right)^{2}\left(c_{2}-1\right)^{2}\left(c_{1} c_{2}+2 c_{2}-2 c_{1}-1\right)\right) .
\end{aligned}
$$

Lemma 2.6 The function $f(x, y)=4(-x+y)^{3}-(x+1)^{2}(y-1)^{2}(x y-2 x+2 y-1)$ is positive on $D=\{(x, y):-1<y<x<1\}$.

Lemma 2.6 and Eq. 2.21 give

$$
\hat{\xi}_{21}\left(x, c_{2} x, z\right)-\hat{\eta}_{2}\left(x, c_{2} x, z\right)>0 .
$$

Now, Eqs. 2.20 and 2.22 imply that similarly as above, the set $\left\{\hat{\eta}_{2}=0\right\} \cap\left\{\hat{\xi}_{21}=0\right\} \cap$ $\left\{-\frac{2 c_{1} c_{2}-c_{1}+3 c_{2}}{-3 c_{1}+c_{2}-2} x<y<E_{4} x\right\}$ is of the form $\left\{y=S_{2} x\right\}$ with $c_{2}<S_{2}<E_{4}$. We deduce that

$$
\hat{J}^{+}(0) \cap\{z \leq 0\}=\hat{A}_{4} \cup \hat{A}_{5} \cup \hat{A}_{6}
$$

where

$$
\begin{aligned}
& \hat{A}_{4}=\left\{\hat{\eta}_{2} \leq 0\right\} \cap\left\{-x \leq y \leq S_{2} x\right\} \cap\{z \leq 0\}, \\
& \hat{A}_{5}=\left\{\hat{\xi}_{21} \leq 0\right\} \cap\left\{S_{2} x \leq y \leq c_{1} x\right\} \cap\{z \leq 0\}, \\
& \hat{A}_{6}=\left\{\hat{\xi}_{22} \leq 0\right\} \cap\left\{c_{1} x \leq y \leq x\right\} \cap\{z \leq 0\} .
\end{aligned}
$$

We conclude this section with the following:

Proposition 2.2 If $W>0$, then

$$
\begin{gathered}
\hat{J}^{+}(0)=\hat{A}_{1} \cup \ldots \cup \hat{A}_{6}, \\
\hat{I}^{+}(0)=\operatorname{int}\left(\hat{A}_{1} \cup \ldots \cup \hat{A}_{6}\right) \cup A_{7} \cup A_{8}
\end{gathered}
$$

and

$N^{+}(0)=\operatorname{int}\left(\hat{A}_{1} \cup \ldots \cup \hat{A}_{6}\right) \cup\left(\left\{\hat{\eta}_{1}=0\right\} \cap\left\{S_{1} x \leq y \leq x\right\}\right) \cup\left(\left\{\hat{\eta}_{2}=0\right\} \cap\left\{-x \leq y \leq S_{2} x\right\}\right)$

where $\hat{A}_{1}, \ldots, \hat{A}_{6}$ are given by Eqs. $2.15-2.17,2.23,2.24$, and 2.25 , respectively, and $A_{7}=$ $\left\{y=c_{1} x, z=0, x \geq 0\right\}, A_{8}=\left\{y=c_{2} x, z=0, x \geq 0\right\}$.

In this way, we see that there are two geometrically optimal timelike (abnormal) curves, and we need six analytic functions to describe reachable sets.

The signs of $z$-coordinates of the fields $\hat{X}+\hat{Y}, \hat{X}-\hat{Y}$, needed in the computation of $\partial \hat{J}^{+}(0) \cap\{z<0\}$, are illustrated in Fig. 3. As above, arrows pointing up (resp. down) correspond to the field $\hat{X}+\hat{Y}(\operatorname{resp} \cdot \hat{X}-\hat{Y})$.

Now, using the notation from the end of Section 2.1, we list all geometrically optimal curves in the case $W>0$. They can be divided into two groups:

(i) Those forming $\partial \hat{J}^{+}(0) \cap\{z \geq 0\}$, i.e. $(\hat{X}+\hat{Y})(\hat{X}-\hat{Y}),\left(\hat{X}+c_{2} \hat{Y}\right)(\hat{X}+\hat{Y})$, $\left(\hat{X}+c_{2} \hat{Y}\right)(\hat{X}-\hat{Y})$; note that $(\hat{X}+\hat{Y})(\hat{X}-\hat{Y})$ and $\left(\hat{X}+c_{2} \hat{Y}\right)(\hat{X}+\hat{Y})$ cease 


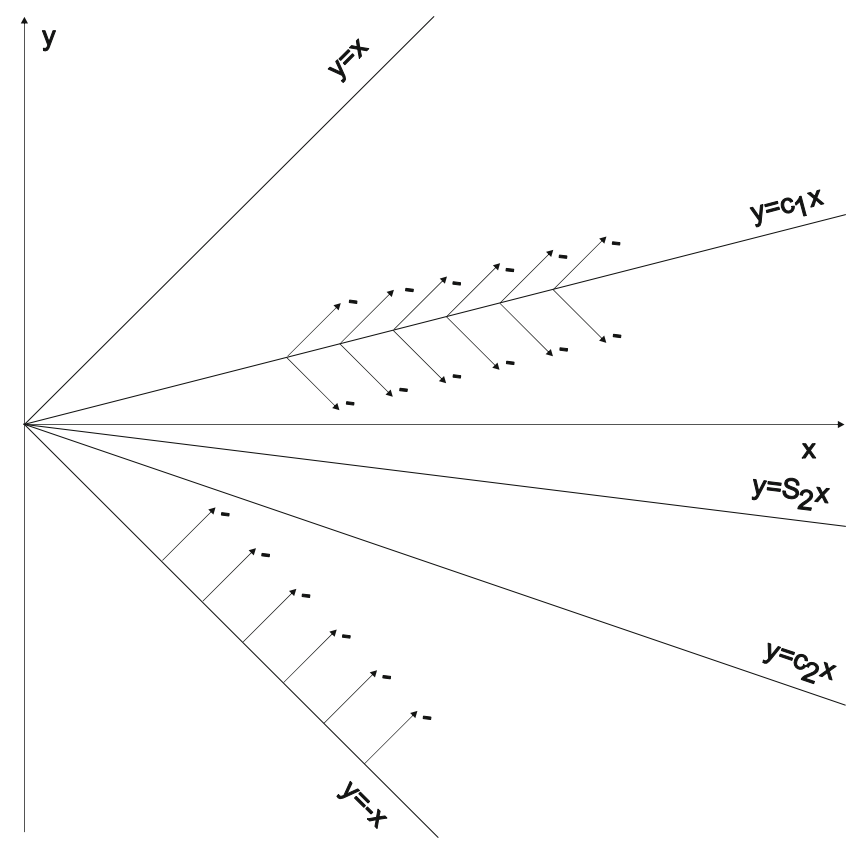

Fig. 3 The case $W>0$. Lines on the plane $\{z=0\}$ along which the fields $\hat{X}+\hat{Y}, \hat{X}-\hat{Y}$ point toward the negative direction of the $z$-axis

to be geometrically optimal when they reach the plane $\left\{y=S_{1} x\right\}$ (then they enter the interior int $\left.\hat{J}^{+}(0)\right)$;

(ii) Those forming $\partial \hat{J}^{+}(0) \cap\{z \leq 0\}$, i.e. $(\hat{X}-\hat{Y})(\hat{X}+\hat{Y}),\left(\hat{X}+c_{1} \hat{Y}\right)(\hat{X}-\hat{Y})$, $\left(\hat{X}+c_{1} \hat{Y}\right)(\hat{X}+\hat{Y})$; note that $\left(\hat{X}+c_{1} \hat{Y}\right)(\hat{X}-\hat{Y})$ and $(\hat{X}-\hat{Y})(\hat{X}+\hat{Y})$ cease to be optimal when they intersect the plane $\left\{y=S_{2} x\right\}$. The intersection of the set $\partial \hat{J}^{+}(0)$ with the plane $\{x=$ const $>0\}$ is represented in Fig. 4.

The points $A, B, C, D$ lie on the plane $\{z=0\} ; A$ and $B$ correspond to halflines $\{y=-x, z=0\}$ and $\{y=x, z=0\}$, while $B$ and $C$ to singular trajectories $\left\{y=c_{2} x, z=0\right\}$ and $\left\{y=c_{1} x, z=0\right\}$, respectively. $D E$ represents the surface formed by the trajectories $(\hat{X}+\hat{Y})(\hat{X}-\hat{Y}), B E$ is the surface made up

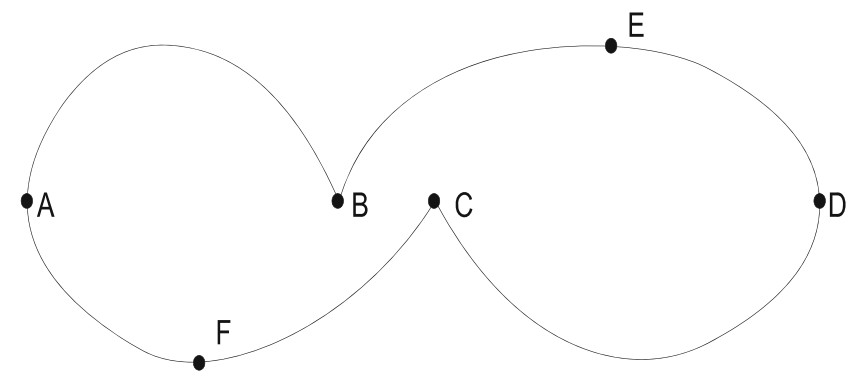

Fig. 4 The set $\partial \hat{J}^{+}(0) \cap\{x=$ const $>0\}$ in the case $W>0$ 
of the trajectories $\left(\hat{X}+c_{2} \hat{Y}\right)(\hat{X}+\hat{Y})$, and the point $E$ corresponds to the set $\partial \hat{J}^{+}(0) \cap\left\{y=S_{1} x\right\}$, i.e., the place where the trajectories $(\hat{X}+\hat{Y})(\hat{X}-\hat{Y})$ and $\left(\hat{X}+c_{2} \hat{Y}\right)(\hat{X}+\hat{Y})$ meet. Finally, part $B A$ of $\partial \hat{J}^{+}(0) \cap\{z \geq 0\}$ is filled with trajectories $\left(\hat{X}+c_{2} \hat{Y}\right)(\hat{X}-\hat{Y})$. Similarly, $A F$ (resp. $C F$ ) is the surface formed by the trajectories $(\hat{X}-\hat{Y})(\hat{X}+\hat{Y})\left(\operatorname{resp} .\left(\hat{X}+c_{1} \hat{Y}\right)(\hat{X}-\hat{Y})\right)$, and $C D$ corresponds to part of $\partial \hat{J}^{+}(0) \cap\{z \leq 0\}$ which is made up of the trajectories $\left(\hat{X}+c_{1} \hat{Y}\right)(\hat{X}+\hat{Y})$. The point $F$ represents the set $\partial \hat{J}^{+}(0) \cap\left\{y=S_{2} x\right\}$, i.e., the place where the trajectories $(\hat{X}-\hat{Y})(\hat{X}+\hat{Y})$ and $\left(\hat{X}+c_{1} \hat{Y}\right)(\hat{X}-\hat{Y})$ meet.

\subsection{The Case $W=0$}

Using Eqs. 2.6-2.9 and the condition $W=0$, we see that in the case under consideration

and in what follows

$$
\begin{gathered}
E_{1}=E_{2}=-\frac{2 c_{1}-1}{c_{1}-2}=c_{2}, \\
E_{3}=E_{4}=\frac{2 c_{2}+1}{c_{2}+2}=c_{1}, \\
\hat{\eta}_{1}(x, y, z)=z+\frac{x^{2}-y^{2}}{12}\left(c_{1}-2\right)\left(y-c_{2} x\right)^{2}, \\
\hat{\eta}_{2}(x, y, z)=-z-\frac{x^{2}-y^{2}}{12}\left(c_{2}+2\right)\left(y-c_{1} x\right)^{2}
\end{gathered}
$$

$$
\begin{gathered}
\hat{X}\left(\hat{\eta}_{1}\right)=\frac{1}{3}(x-y)\left(c_{1}-2\right)\left(y+\frac{2 c_{1}-1}{c_{1}-2} x\right)^{2}=\frac{1}{3}(x-y)\left(c_{1}-2\right)\left(y-c_{2} x\right)^{2}, \\
\hat{X}\left(\hat{\eta}_{2}\right)=-\frac{1}{3}(x+y)\left(c_{2}+2\right)\left(y-\frac{2 c_{2}+1}{c_{2}+2} x\right)^{2}=-\frac{1}{3}(x+y)\left(c_{2}+2\right)\left(y-c_{1} x\right)^{2} .
\end{gathered}
$$

Thus, $\nabla_{\hat{H}} \hat{\eta}_{1}$ is null f.d. on $\{-x<y<x\} \cap\left\{y \neq c_{2} x\right\}$, and $\nabla_{\hat{H}} \hat{\eta}_{2}$ is null f.d. on $\{-x<y<x\} \cap\left\{y \neq c_{1} x\right\}$. We can also see that $\hat{\eta}_{1}\left(x, c_{2} x, 0\right)=\hat{\eta}_{2}\left(x, c_{1} x, 0\right)=0$. Moreover, $\nabla_{\hat{H}} \hat{\xi}_{11}$ is null f.d. for $y<-\frac{2 c_{1} c_{2}-3 c_{1}+c_{2}}{c_{1}-3 c_{2}+2} x$, and as above, we make sure that Eq. 2.14 holds together with the following:

$$
\hat{\xi}_{11}\left(x, c_{2} x, z\right)-\hat{\eta}_{1}\left(x, c_{2} x, z\right)=0 .
$$

Similar reasoning as in Section 2.2 shows that $\hat{\xi}_{11}-\hat{\eta}_{1}$ is nondecreasing along trajectories of $X+Y$ starting at $\left\{y=c_{2} x\right\}$. It follows that $\hat{\xi}_{11} \geq \hat{\eta}_{1}$, and in turn,

$$
\left\{\hat{\xi}_{11} \leq 0\right\} \cap\left\{c_{2} x<y<x\right\} \subset\left\{\hat{\eta}_{1} \leq 0\right\} \cap\left\{c_{2} x<y<x\right\} .
$$

Moreover,

$$
\hat{\xi}_{12}(x, y, z)-\hat{\eta}_{1}(x, y, z)=-\frac{1}{48}(x+y)^{4}\left(c_{2}-1\right)^{2} \frac{W}{\left(c_{2}+1\right)^{2}}=0
$$

which is in fact clear without calculations, since both functions satisfy the same linear differential equation with the same boundary conditions on the hypersurface $\left\{y=c_{2} x\right\}$. Similar reasoning shows that

$$
\left\{\hat{\xi}_{21} \leq 0\right\} \cap\left\{-x<y<c_{1} x\right\} \subset\left\{\hat{\eta}_{2} \leq 0\right\} \cap\left\{-x<y<c_{1} x\right\}
$$


and

$$
\hat{\xi}_{22}(x, y, z)-\hat{\eta}_{2}(x, y, z)=-\frac{1}{48}(x-y)^{4}\left(c_{1}+1\right)^{2} \frac{W}{\left(c_{1}-1\right)^{2}}=0 .
$$

We sum up this subsection with the following:

Proposition 2.3 If $W=0$ then

$$
\hat{J}^{+}(0)=\hat{N}^{+}(0)=\hat{A}_{1} \cup \hat{A}_{2}
$$

and

$$
\hat{I}^{+}(0)=\operatorname{int}\left(\hat{A}_{1} \cup \hat{A}_{2}\right) \cup\left\{y=c_{1} x, x>0\right\} \cup\left\{y=c_{2} x, x>0\right\},
$$

where

$$
\begin{aligned}
& \hat{A}_{1}=\left\{(x, y, z) \in \mathbb{R}^{3}: \hat{\eta}_{1}(x, y, z) \leq 0\right\} \cap\{x \geq 0, z \geq 0\}, \\
& \hat{A}_{2}=\left\{(x, y, z) \in \mathbb{R}^{3}: \hat{\eta}_{2}(x, y, z) \leq 0\right\} \cap\{x \geq 0, z \leq 0\} .
\end{aligned}
$$

As we see, this case is very exceptional as compared to the previous cases with $W \neq 0$. Namely, in spite of the fact that there are two geometrically optimal timelike curves, only two analytic functions suffice for describing reachable sets.

We list all geometrically optimal curves in this case: the curves forming $\partial \hat{J}^{+}(0) \cap$ $\{z \geq 0\}$, i.e., the curves $(\hat{X}+\hat{Y})(\hat{X}-\hat{Y}),\left(\hat{X}+c_{2} \hat{Y}\right)(\hat{X}-\hat{Y})$, and the curves forming $\partial \hat{J}^{+}(0) \cap\{z \leq 0\}$, i.e., $(\hat{X}-\hat{Y})(\hat{X}+\hat{Y}),\left(\hat{X}+c_{1} \hat{Y}\right)(\hat{X}+\hat{Y})$. The set $\partial \hat{J}^{+}(0) \cap$ $\{x=$ const $>0\}$ can be depicted similarly as in Fig. 4 , but this time, the curves $D B$ and $B A$ correspond to trajectories of $\hat{X}-\hat{Y}$, and the curves $A C$ and $C D$ correspond to trajectories of $\hat{X}+\hat{Y}$.

Three-dimensional visualizations of reachable sets studied in this section are presented in Section 7.

\section{Normal Forms}

In this section, we consider more general sub-Lorentzian structures $(H, g)$ than those dealt with in Theorem 1.1. At first, we describe the underlaying distribution $H$. So let $H$ be a rank 2 distribution defined on a neighborhood $U$ of the origin in $\mathbb{R}^{3}$, and let $l_{1}, \ldots, l_{k} \geq 2$ be positive integers. $H$ will be said to satisfy the condition $\left(M_{l_{1}, \ldots, l_{k}}\right)$ if it possesses the following properties:

(i) There exist smooth hypersurfaces $S_{1}, \ldots, S_{k}$ in $U$, such that the intersection $\Gamma=$ $\bigcap_{i=1}^{k} S_{i}$ contains the origin, is smooth 1-dimensional, and transverse to $H$; moreover, for each $q \in \Gamma$ and every $i, j=1, \ldots, k, i \neq j, \operatorname{dim}\left(T_{q} S_{i} \cap T_{q} S_{j}\right)=$ $1, \operatorname{dim}\left(T_{q} S_{i} \cap H_{q}\right)=1$;

(ii) $\quad H$ defines a contact structure on $U \backslash \bigcup_{i=1}^{k} S_{i}$;

(iii) For any fixed $i=1, \ldots, k, H_{q}^{l} \subset H_{q}, l \leq l_{i}, H_{q}^{l_{i}+1}=T_{q} \mathbb{R}^{3}$ on the set $S_{i} \backslash \Gamma$.

(iv) $H_{q}^{l} \subset H_{q}, l \leq l_{1}+\ldots+l_{k}-k+1, H_{q}^{l_{1}+\ldots+l_{k}-k+2}=T_{q} \mathbb{R}^{3}$ for every $q \in \Gamma$.

Now, we choose a Lorentzian metric $g$. We make three assumptions:

(v) For each $i=1, \ldots, k$, the field of directions $S_{i} \ni q \longrightarrow T_{q} S_{i} \cap H_{q}$ is timelike; 
(vi) For every $i, j=1, \ldots, k, i \neq j$, the function $S_{i} \cap S_{j} \ni q \longrightarrow$ $\varangle\left(T_{q} S_{i} \cap H_{q}, T_{q} S_{j} \cap H_{q}\right)$ is constant; and

(vii) The abnormal curves foliating the surfaces $S_{1}, \ldots, S_{k}$ are, up to a change of parameter, t.f.d. Hamiltonian geodesics.

We will say that a sub-Lorentzian structure (or metric) $(H, g)$ is of type $M_{l_{1}, \ldots, l_{k}}$ on $U$ if (i)-(vii) hold on $U$. The set $S=\bigcup_{i=1}^{k} S_{i}$ is again called the Martinet surface for $H$. Note that structures of type $M_{l}$ are exactly generalized Martinet sub-Lorentzian structures studied in [9].

Our aim is to prove the following:

Theorem 3.1 Let $(H, g)$ be a time-oriented analytic sub-Lorentzian structure of type $M_{l_{1}, \ldots, l_{k}}$ defined on a neighborhood $U$ of the origin in $\mathbb{R}^{3}$. Then, provided that $U$ is sufficiently small, there exist analytic coordinates $x, y, z$ on $U$ in which $(H, g)$ has an orthonormal frame in the normal form

$$
\begin{aligned}
& X=\frac{\partial}{\partial x}+y \varphi\left(y \frac{\partial}{\partial x}+x \frac{\partial}{\partial y}\right)+\frac{1}{2} y\left(y-c_{1} x\right)^{l_{1}-1} \ldots\left(y-c_{k} x\right)^{l_{k}-1}(1+\psi) \frac{\partial}{\partial z} \\
& Y=\frac{\partial}{\partial y}-x \varphi\left(y \frac{\partial}{\partial x}+x \frac{\partial}{\partial y}\right)-\frac{1}{2} x\left(y-c_{1} x\right)^{l_{1}-1} \ldots\left(y-c_{k} x\right)^{l_{k}-1}(1+\psi) \frac{\partial}{\partial z}
\end{aligned}
$$

where $X$ is a time orientation, $c_{1}, \ldots, c_{k}$ are constants such that $-1<c_{k}<\ldots<c_{1}<1$, $S_{i}=\left\{y=c_{i} x\right\}, i=1, \ldots, k$, and finally $\varphi, \psi$ are analytic functions on $U$ with $\psi(0,0, z)=$ 0 .

We start with the following result.

Lemma 3.1 Suppose that $(H, g)$ is analytic and satisfies the condition $M_{2, \ldots, 2}$ on a neighborhood $U$ of the origin in $\mathbb{R}^{3}$. Then, provided that $U$ is sufficiently small, there are analytic coordinates $x, y, z$ defined on $U$ in which $(H, g)$ admits an orthonormal frame in the following form

$$
\begin{aligned}
& X=\frac{\partial}{\partial x}-y B\left(y \frac{\partial}{\partial x}+x \frac{\partial}{\partial y}\right)-y\left(y-c_{1} x\right) \ldots\left(y-c_{k} x\right) A \frac{\partial}{\partial z} \\
& Y=\frac{\partial}{\partial y}+x B\left(y \frac{\partial}{\partial x}+x \frac{\partial}{\partial y}\right)+x\left(y-c_{1} x\right) \ldots\left(y-c_{k} x\right) A \frac{\partial}{\partial z}
\end{aligned}
$$

with $X$ being a time orientation. Here, $A, B$ are analytic functions, and $S_{i}=\left\{y=c_{i} x\right\}$, $i=1, \ldots, k$.

Proof Fix an $i, i=1, \ldots, k$. Choose analytic coordinates $\hat{x}, \hat{y}, \hat{z}$ defined in a neighborhood of the origin such that $S_{i}=\{\hat{y}=0\}, \Gamma=\{(0,0, \hat{z})\}, H_{\mid S_{i}}=\operatorname{ker} d \hat{z}$, and $\frac{\partial}{\partial \hat{x}}\left|S_{i}, \frac{\partial}{\partial \hat{y}}\right| S_{i}$ is an orthonormal basis for $H_{\mid S_{i}}$ with a time orientation $\frac{\partial}{\partial \hat{x}}$. Clearly, abnormal curves (which by (vii) are Hamiltonian geodesics) contained in $S_{i}$ satisfy transversality condition with respect to $\Gamma$; cf. [8], Lemma 3.1; see also [2]. Since the satisfaction of the transversality condition does not depend on a choice of coordinates, and, moreover, $i$ was arbitrary, we see that every abnormal curve starting from $\Gamma$ satisfies the transversality condition with respect to $\Gamma$. Now, by use of the exponential mapping and assumption (vii), similarly as it was done 
in $[5,8]$, we are led to the existence of analytic coordinates $x, y, z$ in which $(H, g)$ has an orthonormal frame in the following form:

$$
\begin{aligned}
& X=\frac{\partial}{\partial x}-y B\left(y \frac{\partial}{\partial x}+x \frac{\partial}{\partial y}\right)-y A \frac{\partial}{\partial z} \\
& Y=\frac{\partial}{\partial y}+x B\left(y \frac{\partial}{\partial x}+x \frac{\partial}{\partial y}\right)+x A \frac{\partial}{\partial z}
\end{aligned}
$$

where $S_{j} \cap\{z=0\}=\left\{y=c_{j} x, z=0\right\},\left|c_{j}\right|<1, j=1, \ldots, k, S_{i}=\{y=0\}$ (i.e., $c_{i}=0$ ), and one can suppose that $c_{k}<\ldots<c_{1}$. Using assumption (vi), we see that $S_{j}, j=1, \ldots, k$, are all of the form $S_{j}=\left\{y=c_{j} x\right\}$.

Now, the $z$-coordinate of $[X, Y]$ is equal to

$$
[X, Y](z)=\left(1-y^{2} B\right)\left(A+x \frac{\partial A}{\partial x}\right)-x^{2} y \frac{\partial A}{\partial y} B+x y^{2} \frac{\partial A}{\partial x} B+\left(1+x^{2} B\right)\left(A+y \frac{\partial A}{\partial y}\right) .
$$

By our assumptions $[X, Y]_{\mid y=c_{i} x}$ is horizontal, $i=1, \ldots, k$. Take $i=1$. Then, there exist analytic functions $f(x, z), g(x, z)$ such that $[X, Y]_{\mid y=c_{1} x}=f(x, z) X_{\mid y=c_{1} x}+$ $g(x, z) Y_{\mid y=c_{1} x}$. This leads us to the equality of the $z$-coordinates

$$
2 A+\left(\frac{\partial A}{\partial x}+c_{1} \frac{\partial A}{\partial y}\right) x+\left(1-c^{2}\right) A B x^{2}=\left(g(x, z)-c_{1} f(x, z)\right) x A
$$

where $A$ and its derivatives should be evaluated at $\left(x, c_{1} x, z\right)$. Suppose that $A\left(x, c_{1} x, z\right)$ does not vanish identically. Then, we can find $z$ such that $A\left(x, c_{1} x, z\right)=a_{m}(z) x^{m}+$ $o\left(x^{m}\right)$ as $x \longrightarrow 0$ with $a_{m}(z) \neq 0$ and $m>0$. Since $x\left(\frac{\partial A}{\partial x}+c_{1} \frac{\partial A}{\partial y}\right)_{\mid y=c_{1} x}=$ $x\left(X+c_{1} Y\right)(A)_{\mid y=c_{1} x}=m a_{m}(z) x^{m}+o\left(x^{m}\right)$, Eq. 3.2 gives

$$
(2+m) a_{m}(z)=o(1),
$$

so we arrive at $a_{m}(z)=0$ which is a contradiction. In this way, $A$ may be replaced by the expression $\left(y-c_{1} x\right) A$ for some other analytic function $A$.

Repeating the argument for $i=2, \ldots, k$, we are lead to Eq. 3.1.

Now, suppose that our structure $(H, g)$, which is given by Eq. 3.1 on a neighborhood $U$ of the origin, satisfies the condition $M_{l_{1}, \ldots, l_{k}}, l_{i} \geq 2, i=1, \ldots, k$.

Fix an index $i$. If we set $A_{1}$ to be the function defined by $y\left(y-c_{i} x\right) A_{1}=$ $y\left(y-c_{1} x\right) \ldots\left(y-c_{k} x\right) A$, the frame (3.1) takes the following form:

$$
\begin{aligned}
& X=\frac{\partial}{\partial x}-y B\left(y \frac{\partial}{\partial x}+x \frac{\partial}{\partial y}\right)-y\left(y-c_{i} x\right) A_{1} \frac{\partial}{\partial z} \\
& Y=\frac{\partial}{\partial y}+x B\left(y \frac{\partial}{\partial x}+x \frac{\partial}{\partial y}\right)+x\left(y-c_{i} x\right) A_{1} \frac{\partial}{\partial z}
\end{aligned}
$$

Applying the following change of coordinates

$$
\left\{\begin{array}{l}
\tilde{x}=x \cosh \varphi-y \sinh \varphi \\
\tilde{y}=-x \sinh \varphi+y \cosh \varphi \\
\tilde{z}=z
\end{array},\right.
$$

with $\tanh \varphi=c_{i}$ (i.e., $y-c_{i} x=\tilde{y} / \cosh \varphi$ ) and rewriting (3.3), we are led to

$$
\begin{aligned}
& X=(\cosh \varphi) \tilde{X}-(\sinh \varphi) \tilde{Y} \\
& Y=-(\sinh \varphi) \tilde{X}+(\cosh \varphi) \tilde{Y},
\end{aligned}
$$


where

$$
\begin{aligned}
& \tilde{X}=\frac{\partial}{\partial \tilde{x}}-\tilde{y} B\left(\tilde{y} \frac{\partial}{\partial \tilde{x}}+\tilde{x} \frac{\partial}{\partial \tilde{y}}\right)-\tilde{y}^{2} \tilde{A} \frac{\partial}{\partial \tilde{z}} \\
& \tilde{Y}=\frac{\partial}{\partial \tilde{y}}+\tilde{x} B\left(\tilde{y} \frac{\partial}{\partial \tilde{x}}+\tilde{x} \frac{\partial}{\partial \tilde{y}}\right)+\tilde{x} \tilde{y} \tilde{A} \frac{\partial}{\partial \tilde{z}}
\end{aligned}
$$

and $\tilde{A}=\frac{A_{1}}{\cosh \varphi}$. Obviously (cf. [13]) $\tilde{X}, \tilde{Y}$ is again an orthonormal frame for $(H, g)$ with a time orientation $\tilde{X}$, and we can apply to it the same method as in [9], Proposition 3.2. As a result, Eq. 3.5 can be written as follows:

$$
\begin{aligned}
& \tilde{X}=\frac{\partial}{\partial \tilde{x}}-\tilde{y} B\left(\tilde{y} \frac{\partial}{\partial \tilde{x}}+\tilde{x} \frac{\partial}{\partial \tilde{y}}\right)-\tilde{y}^{l_{i}} \hat{A} \frac{\partial}{\partial \tilde{z}} \\
& \tilde{Y}=\frac{\partial}{\partial \tilde{y}}+\tilde{x} B\left(\tilde{y} \frac{\partial}{\partial \tilde{x}}+\tilde{x} \frac{\partial}{\partial \tilde{y}}\right)+\tilde{x} \tilde{y}^{l_{i}-1} \hat{A} \frac{\partial}{\partial \tilde{z}}
\end{aligned}
$$

with $\tilde{A}=\tilde{y}^{l_{i}-2} \hat{A}$. Passing again to Eq. 3.4, we obtain the following:

$$
\begin{aligned}
& X=\frac{\partial}{\partial x}-y B\left(y \frac{\partial}{\partial x}+x \frac{\partial}{\partial y}\right)-y\left(y-c_{i} x\right)^{l_{i}-1}(\cosh \varphi)^{l_{i}-1} \hat{A} \frac{\partial}{\partial z} \\
& Y=\frac{\partial}{\partial y}+x B\left(y \frac{\partial}{\partial x}+x \frac{\partial}{\partial y}\right)+x\left(y-c_{i} x\right)^{l_{i}-1}(\cosh \varphi)^{l_{i}-1} \hat{A} \frac{\partial}{\partial z}
\end{aligned},
$$

i.e., to say

$$
\begin{aligned}
& X=\frac{\partial}{\partial x}-y B\left(y \frac{\partial}{\partial x}+x \frac{\partial}{\partial y}\right)-y\left(y-c_{1} x\right) \ldots\left(y-c_{i} x\right)^{l_{i}-1} \ldots\left(y-c_{k} x\right) A \frac{\partial}{\partial z} \\
& Y=\frac{\partial}{\partial y}+x B\left(y \frac{\partial}{\partial x}+x \frac{\partial}{\partial y}\right)+x\left(y-c_{1} x\right) \ldots\left(y-c_{i} x\right)^{l_{i}-1} \ldots\left(y-c_{k} x\right) A \frac{\partial}{\partial z}
\end{aligned}
$$

for some new analytic function $A$. Repeating the same argument for every $i=1, \ldots, k$, we are led to

$$
\begin{aligned}
& X=\frac{\partial}{\partial x}-y B\left(y \frac{\partial}{\partial x}+x \frac{\partial}{\partial y}\right)+y\left(y-c_{1} x\right)^{l_{1}-1} \ldots\left(y-c_{k} x\right)^{l_{k}-1} A \frac{\partial}{\partial z} \\
& Y=\frac{\partial}{\partial y}+x B\left(y \frac{\partial}{\partial x}+x \frac{\partial}{\partial y}\right)-x\left(y-c_{1} x\right)^{l_{1}-1} \ldots\left(y-c_{k} x\right)^{l_{k}-1} A \frac{\partial}{\partial z}
\end{aligned}
$$

for yet another analytic $A$ about which we know that does not contain terms of the form $\left(y-c_{i} x\right)^{l}$. Equation 3.6 is, in fact, all we can get without assuming (iv).

Now, we take (iv) into account. To obtain a condition for $A$, we need $l_{1}+\ldots+l_{k}-k+1$ differentiations of $X(z)$ or $Y(z)$, i.e., we have to consider sections of $H^{l_{1}+\ldots+l_{k}-k+2}$. So let $W$ be a (local) section of $H^{l_{1}+\ldots+l_{k}-k+2}$ defined near zero. Then, looking at Eq. 3.6, we see that $W(z)=C A+O(r), r=\sqrt{x^{2}+y^{2}+z^{2}}$, where by (iv) $C \neq 0$ for suitable chosen $W$. Setting $x=y=0$, we arrive at $A(0,0, z) \neq 0$. Last stage is to renormalize the $z$-axis, so as to have $A(0,0, z)=\frac{1}{2}$. This can be done similarly as, e.g., in [9]. To end the proof, we write $\varphi=-B, \psi=2 A-1$.

\section{Reachable Sets in the General Case}

In this section, by $(H, g)$ we denote a fixed time-oriented sub-Lorentzian metric of type $M_{2,2}$, defined on a normal neighborhood $U$ of the origin in $\mathbb{R}^{3}$. Throughout this section, we 
assume that $U$ is as small as we need. We may suppose that $(H, g)$ is already transformed to the normal form. So let $X, Y$ be an orthonormal frame for $(H, g)$ given on $U$ by Eq. 1.1. In cases $W>0, W<0$, we will use the same method to compute local reachable sets as in [7-9]. The mentioned method, however, does not work when $W=0$, and it seems impossible to arbitrate in advance what the structure of the reachable set will be in this case.

Let $X=\hat{X}+X_{1}, Y=\hat{Y}+Y_{2}$, and $\hat{X}, \hat{Y}$ be as in Eq. 2.1, and

$$
\begin{aligned}
& Y_{1}=y \varphi\left(y \frac{\partial}{\partial x}+x \frac{\partial}{\partial y}\right)+\frac{1}{2} y\left(y-c_{1} x\right)\left(y-c_{2} x\right) \psi \frac{\partial}{\partial z} \\
& Y_{2}=-x \varphi\left(y \frac{\partial}{\partial x}+x \frac{\partial}{\partial y}\right)-\frac{1}{2} x\left(y-c_{1} x\right)\left(y-c_{2} x\right) \psi \frac{\partial}{\partial z}
\end{aligned} .
$$

\subsection{The Case $W<0$}

Similarly as in Section 2, consider two Cauchy problems:

$$
(X-Y)(\eta)=0, \quad \eta(x, x, z)=z
$$

with the solution denoted by $\eta_{1}$, and

$$
(X+Y)(\eta)=0, \quad \eta(x,-x, z)=-z
$$

with the solution denoted by $\eta_{2}$. We write $\eta_{1}=\hat{\eta}_{1}+R_{1}, \eta_{2}=\hat{\eta}_{2}+R_{2}$. It is seen that $R_{1}$ and $R_{2}$ satisfy, respectively,

$$
(X-Y)\left(R_{1}\right)=-\left(X_{1}-Y_{1}\right)\left(\hat{\eta}_{1}\right), \quad R_{1}(x, x, z)=0
$$

and

$$
(X+Y)\left(R_{2}\right)=-\left(X_{1}+Y_{1}\right)\left(\hat{\eta}_{2}\right), \quad R_{2}(x,-x, z)=0 .
$$

Clearly, $\left(X_{1}-Y_{1}\right)\left(\hat{\eta}_{1}\right)=O\left(r^{4}\right), r=\sqrt{x^{2}+y^{2}+z^{2}}$. Since $\eta_{1}-z$ is divisible by $x^{2}-y^{2}$ ( $y=-x$ is the trajectory of $X-Y$ starting at $(0,0,0)$ ), we deduce that $R_{1}=O\left(r^{5}\right)$. Similarly, $R_{2}=O\left(r^{5}\right)$ which, in view of $\hat{\eta}_{i}= \pm z+O\left(r^{4}\right)$, means that $\eta_{i}$ may be regarded as a perturbation of $\hat{\eta}_{i}, i=1,2$. Exactly, e.g., as in subsection 4.1 of [9], we prove that $X\left(\eta_{1}\right)$ is divisible by $x-y$. It follows that $\nabla_{H} \eta_{1}=-X\left(\eta_{1}\right)(X-Y)$ where, by using Eq. 2.4, we have

$$
\begin{gathered}
X\left(\eta_{1}\right)=-\frac{1}{12}(x-y)\left(\left(7 c_{1} c_{2}-2 c_{1}-2 c_{2}+1\right) x^{2}+4\left(c_{1} c_{2}-2 c_{1}-2 c_{2}+1\right) x y\right. \\
\left.+\left(c_{1} c_{2}-2 c_{1}-2 c_{2}+7\right) y^{2}+O\left(r^{3}\right)\right) .
\end{gathered}
$$

It follows that $X\left(\eta_{1}\right)<0$ on $U \cap\{-x<y<x\}$, and $\nabla_{H} \eta_{1}$ is null f.d. on $U \cap\{-x<y<x\}$. Similarly,

$$
\begin{gathered}
X\left(\eta_{2}\right)=-\frac{1}{12}(x+y)\left(\left(7 c_{1} c_{2}+2 c_{1}+2 c_{2}+1\right) x^{2}-4\left(c_{1} c_{2}+2 c_{1}+2 c_{2}+1\right) x y\right. \\
\left.+\left(c_{1} c_{2}+2 c_{1}+2 c_{2}+7\right) y^{2}+O\left(r^{3}\right)\right)
\end{gathered}
$$

from which $X\left(\eta_{2}\right)<0$ on $U \cap\{-x<y<x\}$, and hence, $\nabla_{H} \eta_{2}$ is null f.d. on $U \cap$ $\{-x<y<x\}$. We finish the proof of Theorem 1.2 as in the Section 2.1.

Geometrically, optimal trajectories in this case are the same as in the corresponding flat case, with $\hat{X}$ (resp. $\hat{Y}$ ) replaced by $X$ (resp. $Y$ ). Also, reachable sets look similarly; cf. Fig. 1. 


\subsection{The Case $W>0$}

Here, $X\left(\eta_{1}\right)$ and $X\left(\eta_{2}\right)$ are again given by Eqs. 4.2 and 4.3, respectively. This time, however, $X\left(\eta_{1}\right)<0$ on $\left\{\left(E_{1}+\varepsilon\right) x<y<x\right\} \cap U$ and $X\left(\eta_{2}\right)<0$ on $\left\{-x<y<\left(E_{2}-\varepsilon\right) x\right\} \cap U$, where $\varepsilon>0$ will be supposed to be sufficiently small. Next, we define functions $\xi_{11}, \xi_{12}$ as solutions to the following Cauchy problems:

$$
(X+Y)(\eta)=0, \quad \eta\left(x, c_{2} x, z\right)=z
$$

and

$$
(X-Y)(\eta)=0, \quad \eta\left(x, c_{2} x, z\right)=z,
$$

respectively. As above, we write $\xi_{11}=\hat{\xi}_{11}+R_{11}, \xi_{12}=\hat{\xi}_{12}+R_{12}$, where, e.g., $R_{11}$ satisfies $(X+Y)\left(R_{11}\right)=-\left(X_{1}+Y_{1}\right)\left(\hat{\xi}_{11}\right), R_{11}\left(x, c_{2} x, z\right)=0$. It follows that $R_{11}=O\left(r^{5}\right)$, and similarly $R_{12}=O\left(r^{5}\right)$. So again, we may think of $\xi_{1 i}$ as being perturbations of $\hat{\xi}_{1 i}, i=1,2$. Now, since $X+c_{2} Y_{\mid y=c_{2} x}=\frac{\partial}{\partial x}+c_{2} \frac{\partial}{\partial y},\left(X+c_{2} Y\right)\left(\xi_{11}\right)_{\mid y=c_{2} x}=0$ by definition of $\xi_{11}$. But also $(X+Y)\left(\xi_{11}\right)=0$, from which $X\left(\xi_{11}\right)_{\mid y=c_{2} x}=0$, and therefore $X\left(\xi_{11}\right)$ is divisible by $y-c_{2} x$. We prove analogously that also $X\left(\xi_{12}\right)$ is divisible by $y-c_{2} x$. However, this is not enough for our purposes, and we need the following:

Lemma 4.1 $X\left(\xi_{11}\right)$ and $X\left(\xi_{12}\right)$ are divisible by $\left(y-c_{2} x\right)^{2}$.

Proof We prove the first statement. We already know that $X\left(\xi_{11}\right)=\left(y-c_{2} x\right) g$ for an analytic function $g$. Since $[X, X+Y]=0$ on $\left\{y=c_{2} x\right\}$, it follows that $(X+Y)\left(X\left(\xi_{11}\right)\right)=$ $X(X+Y)\left(\xi_{11}\right)=0$ on $\left\{y=c_{2} x\right\}$, where

$$
(X+Y)\left(X\left(\xi_{11}\right)\right)=\left(1-c_{2}-(y-x)\left(c_{2} y+x\right) \varphi\right) g+O\left(\left(y-c_{2} x\right)\right) .
$$

By setting $y=c_{2} x$, we arrive at $\left(1-c_{2}\right)\left[1+\left(c_{2}+1\right) x^{2} \varphi\right] g_{\mid y=c_{2} x}=0$, and the proof is over since $g$ must be divisible by $y-c_{2} x$ (recall that $U$ is as small as we need).

The proof of the second statement is analogous. We notice that $[X, X-Y]=0$ on $\left\{y=c_{2} x\right\}$, so $(X-Y)\left(X\left(\xi_{11}\right)\right)=X(X-Y)\left(\xi_{11}\right)=0$ on $\left\{y=c_{2} x\right\}$ and continue in the same manner.

Making use of Eqs. 2.10, 2.11, and the above lemma, $\nabla_{H} \xi_{11}=-X\left(\xi_{11}\right)(X+Y)$ with

$$
X\left(\xi_{11}\right)=\frac{\left(y-c_{2} x\right)^{2}}{3\left(c_{2}-1\right)^{2}}\left(\left(2 c_{1} c_{2}-3 c_{1}+c_{2}\right) x+\left(c_{1}-3 c_{2}+2\right) y+O\left(r^{2}\right)\right)
$$

and $\nabla_{H} \xi_{12}=-X\left(\xi_{12}\right)(X-Y)$ with

$$
X\left(\xi_{12}\right)=-\frac{\left(y-c_{2} x\right)^{2}}{3\left(c_{2}+1\right)^{2}}\left(\left(2 c_{1} c_{2}+3 c_{1}-c_{2}\right) x+\left(c_{1}-3 c_{2}-2\right) y+O\left(r^{2}\right)\right) .
$$

This, of course, implies that $\nabla_{H} \xi_{11}$ is null f.d. on $\left\{c_{2} x<y<\left(-\frac{2 c_{1} c_{2}-3 c_{1}+c_{2}}{c_{1}-3 c_{2}+2}-\varepsilon\right) x\right\} \cap U$, and $\nabla_{H} \xi_{12}$ is null f.d. on $\left\{-x<y<c_{2} x\right\} \cap U$.

Using just the presented considerations and remembering Section 2.2, we may suppose that $\xi_{11}<\eta_{1}$ on $\left\{c_{2} x<y<\left(E_{1}+\varepsilon\right) x\right\} \cap U$, while $\xi_{11}>\eta_{1}$ on $\left\{\left(c_{1}-\varepsilon\right) x<y<x\right\} \cap U$ or, which is more convenient to us, that $\xi_{11}<\eta_{1}$ on $\left\{c_{2} x<y<\left(S_{1}-\varepsilon\right) x\right\} \cap U$, while $\xi_{11}>\eta_{1}$ on $\left\{\left(S_{1}+\varepsilon\right) x<y<x\right\} \cap U$.

Let us define a set $Z_{1}$ by

$$
Z_{1}=\left\{\eta_{1}=0\right\} \cap\left\{\xi_{11}=0\right\} \cap\left\{\left(S_{1}-\varepsilon\right) x<y<\left(S_{1}+\varepsilon\right) x\right\} \cap U ;
$$


clearly $Z_{1}$ is a semi-analytic set (cf. [12]). As in [8] and [9], we make sure that $\operatorname{dim} Z_{1}=1$ and that $Z_{1}$ is made up of a single analytic curve entering the origin. Further, let us define a semi-analytic set by $\Sigma_{1}=\rho^{-1}\left(\rho\left(Z_{1}\right)\right) \cap U$ where $\rho: \mathbb{R}^{3} \longrightarrow \mathbb{R}^{2}$ is the projection $(x, y, z) \longrightarrow(x, y)$, and let $\Sigma_{1}^{+}, \Sigma_{1}^{-}$be the two connected components of $U \cap\{x \geq 0\} \cap$ $\{z \geq 0\} \cap\left\{c_{2} x \leq y \leq x\right\} \backslash \Sigma_{1}$, containing half-lines $y=x, x \geq 0$ and $y=c_{2} x, x \geq 0$, respectively. All what we have just said leads us to

$$
J^{+}(0, U) \cap\{z \geq 0\}=A_{1} \cup A_{2} \cup A_{3}
$$

where

$$
\begin{gathered}
A_{1}=\left\{\eta_{1} \leq 0\right\} \cap \Sigma_{1}^{+}, \\
A_{2}=\left\{\xi_{11} \leq 0\right\} \cap \Sigma_{1}^{-}, \\
A_{3}=\left\{\xi_{12} \leq 0\right\} \cap\left\{-x \leq y \leq c_{2} x\right\} \cap\{z \geq 0\} \cap U
\end{gathered}
$$

as it was announced in Theorem 1.3.

Quite similar considerations can be carried out to describe the set $J^{+}(0, U) \cap\{z \leq 0\}$. We only note that this time, we define a 1-dimensional semi-analytic set

$$
Z_{2}=\left\{\eta_{2}=0\right\} \cap\left\{\xi_{21}=0\right\} \cap\left\{\left(S_{2}-\varepsilon\right) x<y<\left(S_{2}+\varepsilon\right) x\right\} \cap U .
$$

Then, we set $\Sigma_{2}=\rho^{-1}\left(\rho\left(Z_{2}\right)\right) \cap U$ and define $\Sigma_{2}^{+}, \Sigma_{2}^{-}$to be the two connected components of $U \cap\{x \geq 0\} \cap\{z \leq 0\} \cap\left\{-x \leq y \leq c_{1} x\right\} \backslash \Sigma_{2}$, containing half-lines $y=c_{1} x, x \geq 0$, and $y=-x, x \geq 0$, respectively. Finally, we obtain

$$
J^{+}(0, U) \cap\{z \leq 0\}=A_{4} \cup A_{5} \cup A_{6}
$$

where

$$
\begin{gathered}
A_{4}=\left\{\eta_{2} \leq 0\right\} \cap \Sigma_{2}^{-}, \\
A_{5}=\left\{\xi_{21} \leq 0\right\} \cap \Sigma_{2}^{+}, \\
A_{6}=\left\{\xi_{22} \leq 0\right\} \cap\left\{c_{1} x \leq y \leq x\right\} \cap\{z \leq 0\} \cap U .
\end{gathered}
$$

This terminates the proof of Theorem 1.3.

All geometrically optimal curves are listed in Section 2.2 (again with $\hat{X}, \hat{Y}$ replaced by $X, Y)$, and $\tilde{\partial} J^{+}(0, U) \cap\{x=$ const $>0\}$ can be depicted as in Fig. 4.

\subsection{The Case $W=0$}

Here, as it was mentioned earlier, we are not able to say what the structure of reachable sets is. This is, for instance, because the relation $\hat{\eta}_{1}\left(x, c_{2} x, 0\right)=0$ may no longer be true after perturbation. Therefore, we cannot predict the sign of the expression $\eta_{1}\left(x, c_{2} x, 0\right)$ even in a small neighborhood of the origin without computing higher-order terms in the expression for $\eta_{i}$. We will not do it in this paper.

\subsection{Nilpotent Approximations}

Note that all the functions describing reachable sets in the flat case, i.e., $\hat{\eta}_{i}, \hat{\xi}_{i j}$, and $i, j=$ 1,2 , are homogeneous with respect to the family of dilatations $\delta_{t}(x, y, z)=\left(t x, t y, t^{4} z\right)$. In other words, the mentioned functions are homogeneous when we prescribe the following 
weights to variables: weight $(x)=$ weight $(y)=1$, weight $(z)=4$. It also means that the flat structure is the nilpotent approximation for structures given by Eq. 1.1; cf. [3].

\section{Applications to Control Affine Systems}

Let us consider a control affine system

$$
\dot{q}=X+u Y, \quad u \in[a, b],
$$

defined on a neighborhood $U$ of the origin in $\mathbb{R}^{3}$, where $X$ and $Y$ are supposed to be an orthonormal frame for the sub-Lorentzian metric of type $M_{2,2}$. We can assume that $X$ and $Y$ are given by Eq. 1.1. As it was mentioned in Section 1, the reachable set $\mathcal{A}_{[a, b]}(0, U)$ for Eq. 5.1 coincides with the future nonspacelike reachable set $J^{+}(0, U)$ for the time-oriented sub-Lorentzian structure $\left(H, g^{a, b}\right)$ defined by declaring the frame

$$
\begin{aligned}
& Z^{a, b}=X+\frac{1}{2}(b+a) Y \\
& W^{a, b}=\frac{1}{2}(b-a) Y
\end{aligned}
$$

to be orthonormal with a time orientation $Z^{a, b}$. Thus, $\mathcal{A}_{[a, b]}(0, U)=J^{+}(0, U)$ is just the reachable set for the system

$$
\dot{q}=Z^{a, b}+u W^{a, b}, \quad u \in[-1,1],
$$

(cf. Lemmas 1.1 and 1.2 in [9]).

The aim of this section is to prove Theorem 1.4. To save space, we will not give exact formulas for functions describing reachable sets. We will restrict ourselves only in examining the structure of reachable sets and its dependence on geometric optimality of singular trajectories.

The first evident observation is that

$$
\mathcal{A}_{[a, b]}\left(q_{0}, U\right) \subset\{a x \leq y \leq b x\} \cap U .
$$

\subsection{The Case $c_{1}, c_{2} \notin(a, b)$}

In this case, there are no singular trajectories starting from the origin for Eq. 5.2 (and hence for Eq. 5.1 since both systems are equivalent). As in [7, 9] or as above, we investigate $z$-coordinates of $Z^{a, b} \pm W^{a, b}$. So,

$$
\left(Z^{a, b}+W^{a, b}\right)(z)=\frac{1}{2}\left(y-c_{1} x\right)\left(y-c_{2} x\right)(y-b x)(1+\psi)
$$

and

$$
\left(Z^{a, b}-W^{a, b}\right)(z)=\frac{1}{2}\left(y-c_{1} x\right)\left(y-c_{2} x\right)(y-a x)(1+\psi)
$$

have opposite signs on $\{a x<y<b x\} \cap U$. In this way, again as in [7,9], $\mathcal{A}_{[a, b]}\left(q_{0}, U\right)$ is described by two analytic functions.

\subsection{The Case $c_{2} \leq a<c_{1}<b$ or $a<c_{2}<b \leq c_{1}$}

In this case, the system (5.2) has one singular trajectory initiating at the origin. Again, we examine the signs of $z$-coordinates of $Z^{a, b} \pm W^{a, b}$. Thus, in the first case, (5.4) on $\{y=a x\}$ and Eq. 5.5 on $\{y=b x\}$ are both positive, while in the second case, they are both negative. Also, Eqs. 5.4 and 5.5 are negative near $\left\{y=c_{1} x\right\}$ in the first case, while Eqs. 5.4 and 5.5 
are positive near $\left\{y=c_{2} x\right\}$ in the second case. Therefore, we arrive at the similar situation as in [9], and a similar reasoning as in [9] leads to the conclusion that the minimal number of analytic functions needed for describing $\mathcal{A}_{[a, b]}\left(q_{0}, U\right)$ is four.

\subsection{The Case $a<c_{2}<c_{1}<b$}

In this case, the system (5.2) has two singular trajectories initiating at the origin. In order to simplify the situation, we make the following change of coordinates in Eq. 5.2:

$$
\left\{\begin{array}{l}
\tilde{x}=x \\
\tilde{y}=-\frac{b+a}{b-a} x+\frac{2}{b-a} y . \\
\tilde{z}=z
\end{array} .\right.
$$

The resulting system is as follows:

$$
\dot{q}=\tilde{Z}^{a, b}+u \tilde{W}^{a, b}, \quad u \in[-1,1],
$$

where

$$
\begin{aligned}
& \tilde{Z}^{a, b}=\frac{\partial}{\partial \tilde{x}}+\varphi A^{a, b}+\frac{1}{2}\left(\frac{b-a}{2}\right)^{3} \tilde{y}\left(\tilde{y}-\tilde{c}_{1} \tilde{x}\right)\left(\tilde{y}-\tilde{c}_{2} \tilde{x}\right)(1+\psi) \frac{\partial}{\partial \tilde{z}} \\
& \tilde{W}^{a, b}=\frac{\partial}{\partial \tilde{y}}-\varphi B^{a, b}-\frac{1}{2}\left(\frac{b-a}{2}\right)^{3} \tilde{x}\left(\tilde{y}-\tilde{c}_{1} \tilde{x}\right)\left(\tilde{y}-\tilde{c}_{2} \tilde{x}\right)(1+\psi) \frac{\partial}{\partial \tilde{z}}
\end{aligned}
$$

with

$$
\begin{aligned}
& A^{a, b}=\frac{1}{2} \tilde{y}\left(\frac{1}{2}\left(b^{2}-a^{2}\right) \tilde{x}+\frac{1}{2}(b-a)^{2} \tilde{y}\right) \frac{\partial}{\partial \tilde{x}}+\frac{1}{2} \tilde{y}\left(\frac{1}{2}\left(4-(a+b)^{2}\right) \tilde{x}-\frac{1}{2}\left(b^{2}-a^{2}\right) \tilde{y}\right) \frac{\partial}{\partial \tilde{y}} \\
& B^{a, b}=\frac{1}{2} \tilde{x}\left(\frac{1}{2}\left(b^{2}-a^{2}\right) \tilde{x}+\frac{1}{2}(b-a)^{2} \tilde{y}\right) \frac{\partial}{\partial \tilde{x}}+\frac{1}{2} \tilde{x}\left(\frac{1}{2}\left(4-(a+b)^{2}\right) \tilde{x}-\frac{1}{2}\left(b^{2}-a^{2}\right) \tilde{y}\right) \frac{\partial}{\partial \tilde{y}}
\end{aligned}
$$

and

$$
\tilde{c}_{1}=\frac{2 c_{1}-b-a}{b-a}, \quad \tilde{c}_{2}=\frac{2 c_{2}-b-a}{b-a} .
$$

Note that by rescaling the $\tilde{z}$-axis, we can always get rid of the factor $\left(\frac{b-a}{2}\right)^{3}$, so we no longer take care of it. Since the change of coordinates (5.6) is bi-analytic, transforms straight lines onto straight lines, and preserves geometric optimality of trajectories, the reachable set for Eq. 5.7 is described by the same number of analytic functions as $\mathcal{A}_{[a, b]}\left(q_{0}, U\right)$ and has the same number of geometrically optimal singular trajectories. Now, we repeat the above arguments. Reachable sets for Eq. 5.7 with $\varphi=\psi=0$ in Eq. 5.8 are computed according to Section 2. Reachable sets for Eq. 5.7 with arbitrary $\varphi$ and $\psi$ in Eq. 5.8 are computed according to Section 4 (here, we should remark that although Eq. 5.8 does not coincide with Eq. 1.1, the argument still works since, as one easily checks, functions describing reachable sets for arbitrary $\varphi$ and $\psi$ are again perturbations of functions describing reachable sets for $\varphi=\psi=0)$. To sum up, $\mathcal{A}_{[a, b]}\left(q_{0}, U\right)$ is described by six analytic function whenever $W\left(\tilde{c}_{1}, \tilde{c}_{2}\right)>0$ and by two analytic functions whenever $W\left(\tilde{c}_{1}, \tilde{c}_{2}\right)<0$. We also know that in case $W\left(\tilde{c}_{1}, \tilde{c}_{2}\right)=0$ and $\varphi=\psi=0$, two analytic functions suffice.

In this way the proof of Theorem 1.4 is over.

Remark 5.1 At the end, let us note that the presented method of analysis of reachable sets works for affine control systems induced by any sub-Lorentzian structure described by Theorem 3.1, but because of a large number of constants, it is difficult to state a general theorem. 


\subsection{Two Examples}

To illustrate how the presented methods work in practice, let us consider two examples.

Example 5.1 Suppose that we are interested in the structure of the reachable set from the origin for the following affine control system:

$$
\dot{q}=X+u Y, \quad u \in[-1,4],
$$

where

$$
\begin{aligned}
& X=\frac{\partial}{\partial x}+\frac{1}{2} y(y-2 x)(y-3 x) \frac{\partial}{\partial z} \\
& Y=\frac{\partial}{\partial y}-\frac{1}{2} x(y-2 x)(y-3 x) \frac{\partial}{\partial z}
\end{aligned}
$$

This system is equivalent to the sub-Lorentzian structure $\left(H, g^{-1,4}\right)$ defined by an orthonormal basis $Z^{-1,4}, W^{-1,4}$ with $Z^{-1,4}$ being a time orientation, where

$$
\begin{aligned}
& Z^{-1,4}=\frac{\partial}{\partial x}+\frac{3}{2} \frac{\partial}{\partial y}+\frac{1}{2}\left(y-\frac{3}{2} x\right)(y-2 x)(y-3 x) \frac{\partial}{\partial z} . \\
& W^{-1,4}=\frac{5}{2} \frac{\partial}{\partial y}-\frac{5}{4} x(y-2 x)(y-3 x) \frac{\partial}{\partial z}
\end{aligned}
$$

According to Eq. 5.6, we change coordinates as follows: $\tilde{x}=x, \tilde{y}=-\frac{3}{5} \tilde{x}+\frac{2}{5} \tilde{y}, \tilde{z}=z$, and as a result, we have

$$
\begin{aligned}
& Z^{-1,4}=\frac{\partial}{\partial \tilde{x}}+\frac{1}{2}\left(\frac{5}{2}\right)^{3} \tilde{y}\left(\tilde{y}-\frac{3}{5} \tilde{x}\right)\left(\tilde{y}-\frac{1}{5} \tilde{x}\right) \frac{\partial}{\partial \tilde{z}} \\
& W^{-1,4}=\frac{\partial}{\partial \tilde{y}}-\frac{1}{2}\left(\frac{5}{2}\right)^{3} \tilde{x}\left(\tilde{y}-\frac{3}{5} \tilde{x}\right)\left(\tilde{y}-\frac{1}{5} \tilde{x}\right) \frac{\partial}{\partial \tilde{z}}
\end{aligned}
$$

We end up with $\tilde{c}_{1}=\frac{3}{5}, \tilde{c}_{2}=\frac{1}{5}$. Since $W\left(\frac{3}{5}, \frac{1}{5}\right)<0$, we know that there are no geometrically optimal singular trajectories; hence, the reachable set from the origin for the system that we started with can be described by two analytic functions. $\partial \mathcal{A}_{[-1,4]}(0, \mathbb{R}) \cap$ $\{x=$ const $>0\}$ can be depicted as in Fig. 1, where this time $A$ (resp. $B$ ) corresponds to half-line $\{y=-x, z=0\}$ (resp. $\{y=4 x, z=0\}$ ). An approximate shape of $\mathcal{A}_{[-1,4]}(0, \mathbb{R})$ can be seen in Fig. 5.

Example 5.2 Consider again the system (5.10) where this time

$$
\begin{aligned}
& X=\frac{\partial}{\partial x}+\frac{1}{2} y(y-x)(y-3 x) \frac{\partial}{\partial z} \\
& Y=\frac{\partial}{\partial y}-\frac{1}{2} x(y-x)(y-3 x) \frac{\partial}{\partial z} .
\end{aligned}
$$

According to the above procedure, we pass to the sub-Lorentzian structure induced by an orthonormal frame:

$$
\begin{aligned}
& Z^{-1,4}=\frac{\partial}{\partial x}+\frac{3}{2} \frac{\partial}{\partial y}+\frac{1}{2}(y-x)(y-3 x)\left(y-\frac{3}{2} x\right) \frac{\partial}{\partial z} . \\
& W^{-1,4}=\frac{5}{2} \frac{\partial}{\partial y}-\frac{5}{4} x(y-x)(y-3 x) \frac{\partial}{\partial z}
\end{aligned}
$$

Making again the same change of coordinates, we are led to

$$
\begin{aligned}
& Z^{-1,4}=\frac{\partial}{\partial \tilde{x}}+\frac{1}{2}\left(\frac{5}{2}\right)^{3} \tilde{y}\left(\tilde{y}-\frac{3}{5} \tilde{x}\right)\left(\tilde{y}+\frac{1}{5} \tilde{x}\right) \frac{\partial}{\partial \tilde{z}} \\
& W^{-1,4}=\frac{\partial}{\partial \tilde{y}}-\frac{1}{2}\left(\frac{5}{2}\right)^{3} \tilde{x}\left(\tilde{y}-\frac{3}{5} \tilde{x}\right)\left(\tilde{y}+\frac{1}{5} \tilde{x}\right) \frac{\partial}{\partial \tilde{z}}
\end{aligned},
$$




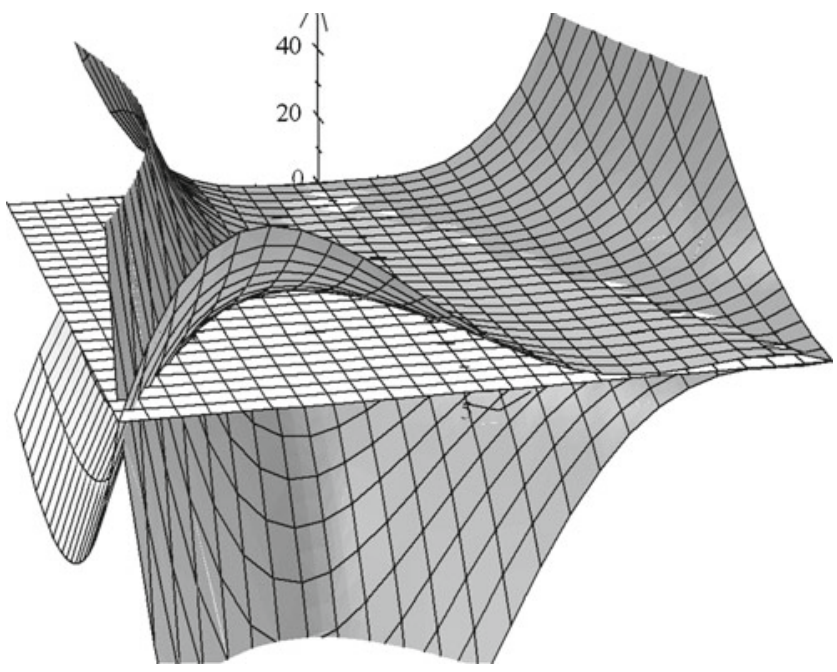

Fig. 5 The surfaces bounding the reachable set in the case $W<0$

so $\tilde{c}_{1}=\frac{3}{5}, \tilde{c}_{2}=-\frac{1}{5}$. But this time, $W\left(\frac{3}{5},-\frac{1}{5}\right)>0$; therefore, we conclude that there are two geometrically optimal singular trajectories, and that we need six analytic functions to describe the reachable set $\mathcal{A}_{[-1,4]}(0, \mathbb{R})$ for the system we started with. The intersection $\partial \mathcal{A}_{[-1,4]}(0, \mathbb{R}) \cap\{x=$ const $>0\}$ can be represented, with obvious changes in the interpretation, as in Fig. 4. $\mathcal{A}_{[-1,4]}(0, \mathbb{R})$ looks approximately as in Fig. 7.

Remark 5.2 Let us notice in this place that the structure of reachable sets in the case $a<$ $c_{2}<c_{1}<b$ essentially depends on $a$ and $b$. For instance, if in the last example with $c_{1}=3$, $c_{2}=1, a=-1$, we let $b$ vary then (cf. 5.9), the expression $W\left(\frac{2 c_{1}-b-a}{b-a}, \frac{2 c_{2}-b-a}{b-a}\right)=$ $W\left(\frac{6-b+1}{b+1}, \frac{2-b+1}{b+1}\right)=-4 \frac{b-7}{(b+1)^{2}}$ changes sign when $b$ exceeds 7 .

\section{Proofs of Lemmas from Section 2.2}

In this section, we present the proofs of lemmas from Section 2.

Proof of Lemma 2.1 The proof relies on straightforward computations. For instance, $E_{1}<1$ is equivalent to

$$
\sqrt{-3\left(c_{1} c_{2}-2 c_{1}+2 c_{2}-1\right)\left(c_{1} c_{2}+2 c_{1}-2 c_{2}-1\right)}<3 c_{1} c_{2}-6 c_{1}-6 c_{2}+9,
$$

where $3 c_{1} c_{2}-6 c_{1}-6 c_{2}+9=3\left(W+4-4 c_{1}\right)$ is positive. Squaring both sides of Eq. 6.1, we see that Eq. 6.1 is equivalent to

$$
12\left(c_{2}-1\right)\left(c_{1}-1\right)\left(c_{1} c_{2}-2 c_{1}-2 c_{2}+7\right)>0
$$

which is, of course, true. The remaining inequalities are proved analogously.

Proof of Lemma 2.2 We prove, for instance, that $c_{2}<E_{4}$. By Eq. 2.9, this is equivalent to $-\left(c_{2}+2\right)\left(2 c_{2}+c_{1} c_{2}-1-2 c_{1}\right)>\sqrt{-3\left(c_{1} c_{2}-2 c_{1}+2 c_{2}-1\right)\left(c_{1} c_{2}+2 c_{1}-2 c_{2}-1\right)}$ 
which, in turn, is equivalent to

$$
\begin{aligned}
& \left(c_{2}+2\right)^{2}\left(2 c_{2}+c_{1} c_{2}-1-2 c_{1}\right)^{2}+3\left(c_{1} c_{2}-2 c_{1}+2 c_{2}-1\right)\left(c_{1} c_{2}+2 c_{1}-2 c_{2}-1\right) \\
= & \left(c_{2}-1\right)\left(c_{2}+1\right)\left(c_{1} c_{2}+2 c_{1}+2 c_{2}+7\right)\left(2 c_{2}+c_{1} c_{2}-1-2 c_{1}\right)>0 .
\end{aligned}
$$

Proof of Lemma 2.3 We will prove the first inequality. Since $c_{1}-3 c_{2}+2>0$, the hypothesis is equivalent to $-\left(2 c_{1} c_{2}-3 c_{1}+c_{2}\right)-c_{1}\left(c_{1}-3 c_{2}+2\right)=\left(1-c_{1}\right)\left(c_{1}-c_{2}\right)>0$ which is, of course, true.

Proof of Lemma 2.4 We look for stationary points of $f$. Any such point $(x, y)$ must satisfy the equality

$$
\frac{\partial f}{\partial x}(x, y)+\frac{\partial f}{\partial y}(x, y)=(x+1)(y-1)(x+y)(3 x y+5 y-5 x-3)=0 .
$$

Clearly, $3 x y+5 y-5 x-3=3(x y-1)-5(x-y)<0$ in $D$, thus, either $x=-1$ or $y=1$, or $y=-x$. If $y=-x$ in $D$, we must have $x>0$, but then $\frac{\partial f}{\partial x}(x,-x)=$ $-3 x^{5}-20 x^{4}-46 x^{3}-23 x-4<0$, while $\frac{\partial f}{\partial y}(x,-x)=3 x^{5}+20 x^{4}+46 x^{3}+23 x+4>0$. It follows that all stationary points of $f$ are contained in $\partial D$. By direct calculation, we make sure that $f_{\mid \partial D} \leq 0$ which implies $f<0$ in $D$.

Proof of Lemma 2.5 We prove the second inequality. Since $-3 c_{1}+c_{2}-2<0$, the hypothesis is equivalent to $-\left(2 c_{1} c_{2}-c_{1}+3 c_{2}\right)-c_{2}\left(-3 c_{1}+c_{2}-2\right)=\left(c_{2}+1\right)\left(c_{1}-c_{2}\right)>0$.

Proof of Lemma 2.6 This is proved analogously as Lemma 2.4.

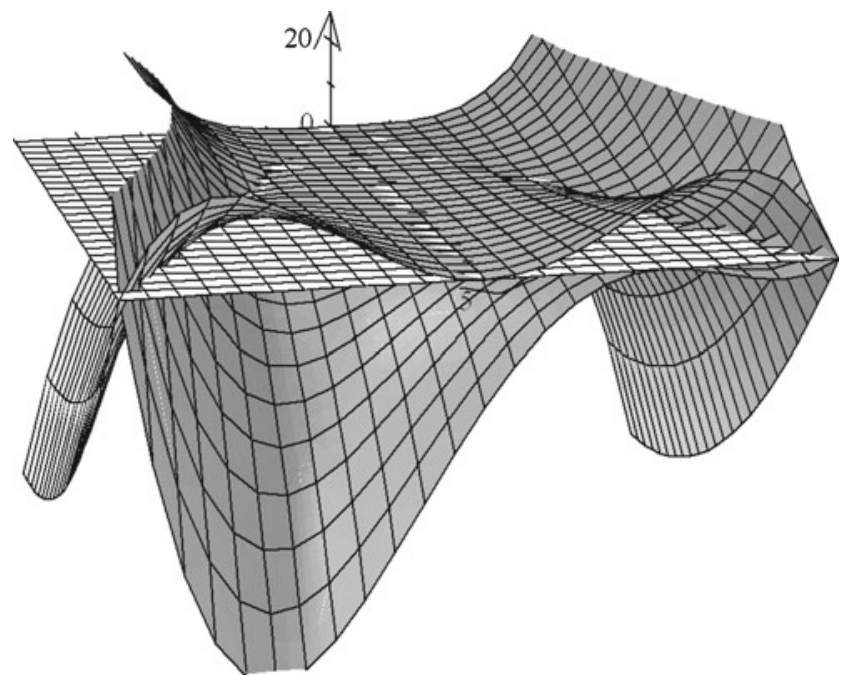

Fig. 6 The surfaces bounding the reachable set in the case $W=0$ 


\section{Pictures}

In this section, we present 3-dimensional visualizations of exemplary reachable sets studied in Section 2.

At first, consider the case $c_{1}=\frac{3}{4}, c_{2}=\frac{1}{2}$. Clearly, $W\left(\frac{3}{4}, \frac{1}{2}\right)=-\frac{1}{8}$, and in this case,

$$
\begin{aligned}
& \hat{\eta}_{1}(x, y, z)=-\frac{1}{128}(x-y)(x+y)\left(-12 x y+3 x^{2}+13 y^{2}\right)+z, \\
& \hat{\eta}_{2}(x, y, z)=-\frac{1}{384}(x-y)(x+y)\left(-124 x y+49 x^{2}+79 y^{2}\right)-z .
\end{aligned}
$$

On all the following three figures, the plane $\{z=0\}$ is marked with white color. The corresponding reachable set is bounded by two hypersurfaces: by $\left\{\hat{\eta}_{1}=0\right\}$ from above (darker color) and by $\left\{\hat{\eta}_{2}=0\right\}$ from below (lighter color) as it is presented in Fig. 5.

Next, consider the case $c_{1}=\frac{1}{2}, c_{2}=0$. Now, $W\left(\frac{1}{2}, 0\right)=0$, and

$$
\begin{aligned}
& \hat{\eta}_{1}(x, y, z)=-\frac{1}{8} y^{2}(x-y)(x+y)+z, \\
& \hat{\eta}_{2}(x, y, z)=-\frac{1}{24}(x-y)(x+y)(x-2 y)^{2}-z .
\end{aligned}
$$

The reachable set in this case is presented in Fig. 6. As in the previous case, it is the set bounded by two hypersurfaces $\left\{\hat{\eta}_{i}=0\right\}, i=1,2$, with similarly chosen colors.

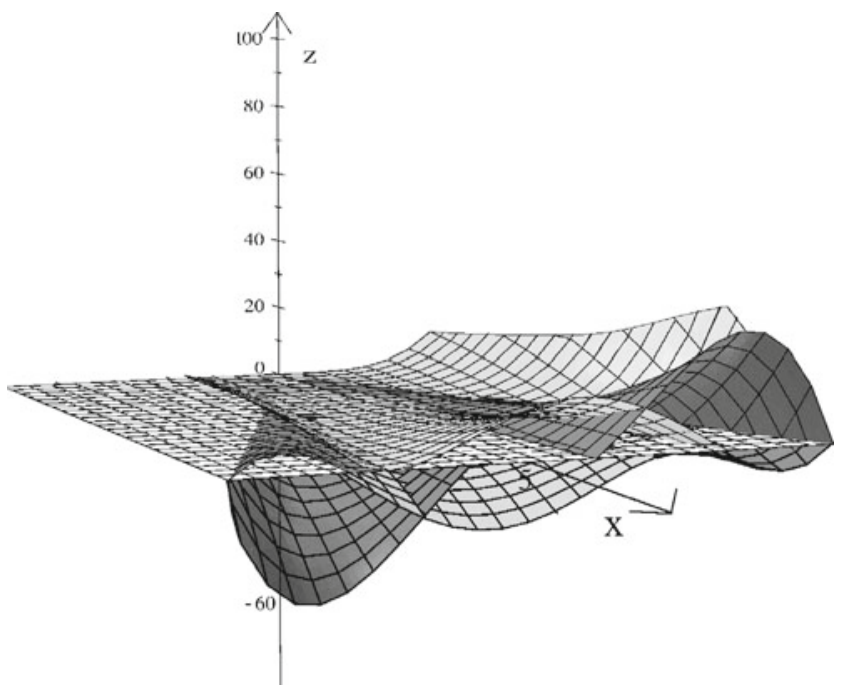

Fig. 7 The surfaces bounding the reachable set in the case $W>0$ 
Finally, consider the case $c_{1}=\frac{1}{2}, c_{2}=-\frac{1}{2}$. This time $W\left(\frac{1}{2},-\frac{1}{2}\right)=\frac{3}{4}$, and

$$
\begin{aligned}
& \hat{\eta}_{1}(x, y, z)=\frac{1}{64}(x-y)(x+y)\left(-4 x y+x^{2}-9 y^{2}\right)+z, \\
& \hat{\eta}_{2}(x, y, z)=\frac{1}{64}(x-y)(x+y)\left(4 x y+x^{2}-9 y^{2}\right)-z, \\
& \hat{\xi}_{11}(x, y, z)=-\frac{1}{216}(5 x-8 y)(x-y)(x+2 y)^{2}+z, \\
& \hat{\xi}_{12}(x, y, z)=-\frac{1}{8} x(x+y)(x+2 y)^{2}+z, \\
& \hat{\eta}_{2}(x, y, z)=\frac{1}{64}(x-y)(x+y)\left(4 x y+x^{2}-9 y^{2}\right)-z, \\
& \hat{\xi}_{21}(x, y, z)=-\frac{1}{216}(x+y)(5 x+8 y)(x-2 y)^{2}-z, \\
& \hat{\xi}_{22}(x, y, z)=-\frac{1}{8} x(x-y)(x-2 y)^{2}-z .
\end{aligned}
$$

The corresponding reachable set is presented in Fig. 7. It is the set which is bounded by six hypersurfaces $\left\{\hat{\eta}_{i}=0\right\},\left\{\hat{\xi}_{i j}=0\right\}, i, j=1,2$.

Acknowledgments This work was partially supported by the Polish Ministry of Research and Higher Education, grant NN201 607540. This paper was written during my stay in the Institut Mittag-Leffler in autumn 2011.

\section{Appendix}

In this section, we state some corollaries concerning sub-Lorentzian metrics of type $M_{2,2}$. We start from definitions; cf. [8, 9].

Let $(M, H, g)$ be a sub-Lorentzian manifold. Fix a point $q_{0}$. Denote by $\mathcal{D}_{q_{0}}$, the set of all covectors $\lambda \in T_{q_{0}}^{*} M$ such that the curve $t \longrightarrow \Phi^{t}(\lambda)$ is defined on the whole interval [0,1]. Here, $\Phi^{t}$ stands for the (local) flow of the Hamiltonian vector field $\overrightarrow{\mathcal{H}}$. The exponential mapping $\exp _{q_{0}}: \mathcal{D}_{q_{0}} \longrightarrow M$ is defined to be $\exp _{q_{0}}(\lambda)=\pi \circ \Phi^{1}(\lambda)$, where $\pi: T^{*} M \longrightarrow$ $M$ is the canonical projection. It is seen that if $\gamma(t)$ is the Hamiltonian geodesic with initial condition $\lambda \in T_{q_{0}}^{*} M$, then $\gamma(t)=\exp _{q_{0}}(t \lambda)$. Now, we say that a point $q$ is conjugate to $q_{0}$ if there exists a covector $\lambda \in T_{q_{0}}^{*} M$ such that $\exp _{q_{0}}(\lambda)=q$ and $d_{\lambda} \exp _{q_{0}}$ is singular; in such a case, we say that $q$ is conjugate to $q_{0}$ along the geodesic $t \longrightarrow \exp _{q_{0}}(t \lambda)$. The future timelike (nonspacelike, null) conjugate locus of a point $q_{0}$ is defined to be the set of all points $q$ that are conjugate to $q_{0}$ along the timelike (nonspacelike, null) f.d. Hamiltonian geodesics; it is denoted by Conj $q_{0}^{t}\left(\right.$ Conj $_{0}^{n s p c}$, Conj $\left._{0}^{\text {null }}\right)$. Finally, by the future null cut locus of $q_{0}$, $\mathrm{Cut}_{q_{0}}^{\text {null }}(M)$, we mean the set of points $q \in M$ such that there exists a null f.d. (not necessarily Hamiltonian) geodesic $\gamma:[0, T] \longrightarrow M$ having the following properties: $\gamma(0)=q_{0}$, $\gamma\left(t_{1}\right)=q$ with $0<t_{1}<T, \gamma_{\mid\left[0, t_{1}\right]}$ is a length maximizer and $\gamma_{\mid\left[0, t_{1}+\varepsilon\right]}$ is not a length maximizer for an $\varepsilon>0, t_{1}+\varepsilon \leq T$.

Now, let $(H, g)$ be a sub-Lorentzian structure of type $M_{2,2}$ defined on a normal neighborhood $U$ of $0 \in \mathbb{R}^{3}$. Suppose that $(H, g)$ is given by Eq. 1.1 . 
Below, we list some properties of $\exp _{0}, \operatorname{Conj}_{0}^{t}$, $\operatorname{Conj}_{0}^{\text {null }}$, and $\operatorname{Cut}_{0}^{\text {null }}(U)$. Proofs are omitted since they are similar to those found in $[8,9]$.

\section{A.1 Image Under the Exponential Mapping}

\section{Proposition 8.1}

$$
\exp _{0}\left(\left\{\lambda \in \mathcal{D}_{0}: \mathcal{H}(\lambda)<0,\langle\lambda, X(0)\rangle<0\right\}\right)=I^{+}(0, U),
$$

$\exp _{0}\left(\left\{\lambda \in \mathcal{D}_{0}: \mathcal{H}(\lambda) \leq 0,\langle\lambda, X(0)\rangle<0\right\}\right)=I^{+}(0, U) \cup\{y= \pm x, z=0\} \cap U$.

\section{A.2 Conjugate Locus}

Proposition 8.2 Conj $j_{0}^{\text {null }}$ is equal to the union of the two null f.d. Hamiltonian geodesics starting at 0 , i.e., Con $j_{0}^{\text {null }}=\{y= \pm x, z=0\} \cap U$.

Note that in case $W \leq 0$, the future timelike conjugate locus $\operatorname{Conj}_{0}^{t}$ contains the two abnormal curves starting from 0 (because they are timelike and lie on $\tilde{\partial} J^{+}(0, U)$ ). These curves are unique maximizers.

\section{A.3 Future Null Cut Locus}

Proposition 8.3 Suppose that $W>0$. Then, $C u t_{0}^{\text {null }}(U)=\{0\}$.

Proposition 8.4 Suppose that $W<0$. Then, $C u t_{0}^{\text {null }}(U)=\tilde{\partial} J(0, U) \cap\left(\Sigma_{1} \cup \Sigma_{2}\right)$.

Proposition 8.5 Suppose that $W=0$. Then, $C u t_{0}^{\text {null }}(U)=\left(\left\{y=c_{1} x, z=0\right\} \cup\right.$ $\left.\left\{y=c_{2} x, z=0\right\}\right) \cap U$.

Note that $\operatorname{Cut}_{0}^{\text {null }}(U) \cap \operatorname{Conj}_{0}^{t} \neq \varnothing$ in the case $W=0$.

\section{References}

1. Agrachev A, Sachkov Y. Control theory from geometric viewpoint. Encyclopedia of Mathematical Science. vol. 87. New York: Springer; 2004.

2. Agrachev A, Chakir El-A El-H, Gauthier JP. Sub-Riemannian metrics on $\mathbb{R}^{3}$. Canadian Mathematical Society Conference Proceedings, Vol. 25; 1998.

3. Bellaïche A. The tangent space in the sub-Riemannian geometry. Sub-Riemannian Geometry. Cambridge: Birkhäuser; 1996

4. Bonnard B, Chyba M. Singular trajectories and their role in control theory. Berlin: Springer; 2003.

5. Grochowski M. Normal forms of germs of contact sub-Lorentzian structures on $\mathbb{R}^{3}$. Differentiability of the sub-Lorentzian distance. J Dyn Control Syst. 2003;9(4):531-547.

6. Grochowski M. Properties of reachable sets in the sub-Lorentzian geometry. J Geom Phys. 2009;59:885900 .

7. Grochowski M. Reachable sets for contact sub-Lorentzian structures on $\mathbb{R}^{3}$. Application to control affine systems on $\mathbb{R}^{3}$ with a scalar input. J Math Sci. 2011a;177(3):383-394.

8. Grochowski M. Normal forms and reachable sets for analytic martinet sub-Lorentzian structures of Hamiltonian type. J Dyn Control Syst. 2011b;17(1).

9. Grochowski M. The structure $\mathrm{f}$ reachable sets for affine control systems induced by generalized Martinet sub-Lorentzian metrics. ESAIM: COCV;18:1150-1177. doi:10.1051/cocv/2011202. 
10. Korolko A, Markina I. Nonholonomic Lorentzian geometry on some HI-type groups. J Geom Anal. 2009;19(4):864-889.

11. Liu W, Sussmann H. Shortest paths for sub-Riemannian metrics on rank-two distributions. Mem Am Math Soc. 1995;118(564):x+104.

12. Łojasiewicz S. Ensembles semi-analytiques. Bures-sur-Yvette: Institut des Hautes études Scientifiques; 1964.

13. O’Neill B. Semi-Riemannain geometry with applications to relativity, Pure and Applied Series. vol. 103. New York: Academic; 1983. 